\title{
Rotation of a multi-species plasma in a cylinder
}

\author{
D. Sünder, H. Wobig \\ Max-Planck-Institut für Plasmaphysik, Garching bei München, EURATOM-Ass.
}

\begin{abstract}
The paper investigates the momentum balance of a multi-species plasma in a cylinder with helical magnetic field. The main magnetic field is oriented in the z-direction. Starting from the macrocopic equations, a steady-state solution is obtained which includes the effect of centrifugal forces and Coriolis forces. The poloidal rotation and axial flow of each particle species is governed by the balance between spin-up, viscous damping, turbulent forces and the $\boldsymbol{v} \times \boldsymbol{B}$ driving term. In cylindrical geometry, classical viscous effects are very small and can easily be dominated by the turbulent viscosity. The effect of turbulent Reynolds stresses and the turbulent viscosity is investigated. Under quite general assumptions about the dependence of the anomalous transport coefficients on the velocity shear, criteria on the existence of the bifurcation points and multiple solution can be formulated. The shaping of the velocity shear and the transport barrier depend strongly on the eddy viscosity. Numerical solutions of the differential equations for a two-component plasma support the analytical results. The viscosity is considered as a variable parameter simulating the effect of turbulent eddy viscosity. These computations will be compared with the experimental results found in the HDH-mode experiments in Wendelstein 7-AS.
\end{abstract}

\section{Introduction}

Plasma rotation during L-H-transition is one of the most interesting issues in toroidally confined plasma, since it has been experimentally found that improvement of plasma confinement is accompanied by onset of rotation. Many theories have been developed in order to explain this phenomenon; many of them invoke the bifurcation of electric fields as one of the key elements in LH-transition. Hinton and Staebler [1] proposed a cylindrical model where the anomalous transport coefficients non-linearily depend on the velocity shear allowing for bifurcating solutions of the transport equations. This model has been succesfully applied to DIII-D where the Hmode density barrier can be understood on the basis of this theory [2]. In a quiescent and stable plasma, neoclassical transport exhibits a non-linear dependence on the radial electric field which gives rise to multiple solutions and bifurcations. The wide spectrum of theories concerning LH-transition has been described in the review paper by Connor and Wilson [3].

A major problem in investigating this phenomenon theoretically is the toroidal geometry which makes a mathematical treatment difficult and forces one to make truncations and approximations of the non-linear momentum balance equations and the equations of continuity. In particular, when impurity ions and the inward diffusion of these contribute to the momentum balance a system of coupled differential equations must be handled. Magnetic pumping is the result of viscous dissipation and in this respect tokamaks and stellarators strongly differ. In tokamaks only the poloidal asymmetry leads to magnetic pumping while in stellarators the joint effect of poloidal and toroidal asymmetries cause resonance effects and a strong dependence on the rotational transform [4],[5]. In a torus, the inertial forces, which are the key element in the Stringer spin-up [6], provide a coupling between the poloidal and toroidal rotation which has its source in the Coriolis force. In the physics of the atmosphere, Coriolis forces play a large role 
in the formation of cyclones and hurricanes [7]. Also, Rossby waves, which often are invoked as a paradigm of drift waves are governed by Coriolis forces.

In general toroidal geometry, and in particlar in view of stellarator geometry, this role of inertial forces has been investigated in [8] including the effect of anomalous transport. Thus, in toroidal geometry there are at least three candidates for driving poloidal and toroidal rotation: Stringer spin-up, lost orbits and their recoil effect on the plasma and turbulent Reynolds stresses. Reynolds stresses drive zonal flow which provide a feedback on the turbulence and reduce anomalous transport [9] [10].

In this respect a cylindrical geometry offers some advantages since due to the inherent symmetry several effects can be excluded. In such a cylindrical model, there are no poloidal asymmetries, no geodesic curvature, no Stringer spin-up, no Pfirsch-Schlüter currents, no banana orbits and no neoclassical effects. However, in cylinder geometry the plasma can grow unstable by gradient driven drift waves leading to zonal flow and anomalous transport. The frequently used models of drift-wave turbulence, the Hasegawa-Mima equation [11] and the Hasegawa-Wakatani equations are written in slab geometry. Several models of LH-transition are treated in slab or cylinder geometry. To a large extent cylindrical geometry can be handled without dubious orderings and approximations, which does not mean that no approximations are needed.

In our cylindrical model we should like to distinguish between tokamaks and stellarators. A tokamak has a toroidal current which produces a poloidal field and helical magnetic field lines while in stellarators without toroidal current we may neglect the poloidal field and restrict the magnetic field to an axial field.

This paper is motivated by the discovery of the HDH-mode in Wendelstein 7-AS [12][13][14] [15] where the density profile changes from a peaked profile to a flat profile in the center and strong gradients at the boundary while the temperature profile stays nearly unchanged. In the $\mathrm{HDH}$-mode, the confinement time has increased significantly. Apparently the energy balance does not play a significant role during this transition and for this reason the energy balance has been dropped in the following analysis. The temperature is considered as a given function in the analysis; the numerical computation is restricted to the density profile and the radial elecric field. The equations underlying these computations may be considered as a modified HintonStaebler model. However, in this paper an attempt will be made to present a more rigorous derivation starting from the momentum balance and the equations of continuity of a multispecies plasma. The cylindrical model cannot explain why the transition into the HDH-regime only occurs in specific regimes of the rotational transform. This effect can be attributed to the strong dependenc of viscous damping on rotational transform and magnetic islands, which is a clear toroidal effect [5].

The paper is organized as follows. In section 2 we formulate the momentum balance equations for a multi-species plasma and discuss the dissipative processes and in section 2.1 the linear theory of resistive drift waves is formulated. The spin-up equations of poloidal and toroidal flow on magnetic surfaces will be formulated in general toroidal geometry employing the Hamada coordinate system (section 2.2). The Reynolds averaged equations (section 2.4) include the effect of turbulence and in section 4 a closure of the equations is proposed. Applying these equations to a cylindrical plasma in a helical magnetic field leads to a coupled system of differential equations for the poloidal velicities and axial velocities. Conditions of bifurcation are treated in section 4.1. The radial force balance allows one to compute the density profile and the radial electric field. In section 6 the numerical results of a two-component plasma will be presented. These computations in section 6 simulate the high density regime (HDH-regime) found in the Wendelstein 7-AS stellarator. 


\section{The momentum balance}

The starting point of our analysis is a turbulent plasma which has become unstable due to electrostatic modes - resistive drift modes, resistive interchange modes or ITG-modes etc. We neglect magnetic fluctuations which may be justifield in a low-beta plasma. The turbulence has grown to a finite level of fluctuation and the resulting anomalous plasma losses are balanced by finite source terms maintaining the plasma gradients and the stationary turbulence. We assume zero momentum source to the plasma thus avoiding a situation where plasma rotation is excited externally. Such a model applies to ECR-heated plasmas or plasmas with balanced NB-injection. The momentum balance equations of the multi-fluid model are

$$
\frac{\partial}{\partial t} m_{k} n_{k} \boldsymbol{v}_{k}+\nabla \cdot \mathbf{S}_{k}=q_{k} n_{k}\left(\boldsymbol{E}+\boldsymbol{v}_{k} \times \boldsymbol{B}\right)+\boldsymbol{F}_{k} \quad ; \quad k=1, \ldots K
$$

Here $k$ is the index of the particle species with charge $q_{k}$ and $K$ is the number of charged states. The index $k=K$ denotes the electron equation. In a hydrogen plasma $K$ is 2 , in a fusion plasma $K$ is 4 . In a plasma with impurity ions this number may be rather large since $k$ runs over various impurity species and over the ionization stages of the impurities. The stress tensor is $\mathbf{S}_{k}=p_{k} \mathbf{I}+\pi_{k}+m_{k} n_{k} \boldsymbol{v}_{k}: \boldsymbol{v}_{k} . p_{k}$ is the scalar pressure, $\pi_{k}$ the anisotropic component of the pressure tensor and $m_{k} n_{k} \boldsymbol{v}_{k}: \boldsymbol{v}_{k}$ is the inertial component of the stress tensor. Scalar pressure and density are linked by the ideal gas law $p_{k}=n_{k} T_{k} . \boldsymbol{F}_{k}$ is the friction force caused by Coulomb interaction between charged particles [16]. They are linear functions of the velocities

$$
\boldsymbol{F}_{k}=\sum_{i} l_{11}^{k i} \mathbf{v}_{i} \quad ; \quad l_{11}^{k i}=a_{k i}-\sum_{j} a_{i j} \delta_{i k} \quad ; \quad a_{i k}=n_{i} m_{i k} \nu_{i k}=a_{k i} \quad ; \quad l_{11}^{k i}=l_{11}^{i k}
$$

and because of the momentum conservation of Coulomb forces the sum over all friction forces is zero $\left(\sum_{k} \boldsymbol{F}_{k}=0\right)$. The positive coefficients $a_{i k}$ are proportional to the collision frequencies and symmetric. $m_{i k}$ is the reduced mass. The collision frequencies $\nu_{i k}$ are continuous and bounded if the density and the temperature are continuous and bounded. A more exact expression of the friction force contains the heat fluxes $\boldsymbol{q}$, however the linear dependence on the heat flux vector will not be included here since this would imply including the energy balance and the heat flux equations. The terms $\nabla \cdot \pi_{k}$ are the viscous forces which in the collisional regime are described by the Braginskii viscosity [17] and in the long-mean free path regime by the neoclassical viscosity. The Braginskii viscous operator is a differential operator on the velocity $\boldsymbol{v}_{k}$, however, later theories have added another term depending on the heat flux [19][48][21]. Together with the equation of continuity

$$
\frac{\partial n_{k}}{\partial t}+\nabla n_{k} \boldsymbol{v}_{k}=S_{k}
$$

the force balance can also be written

$$
\begin{gathered}
\frac{\partial \boldsymbol{v}_{k}}{\partial t}+\frac{1}{2} \nabla \boldsymbol{v}_{k}^{2}-\boldsymbol{v}_{k} \times\left(\vec{\omega}_{k}+\vec{\Omega}_{k}\right)+S_{k} \boldsymbol{v}_{k}=-\frac{1}{m_{k}}\left(\frac{\nabla p_{k}}{n_{k}}+q_{k} \nabla \Phi\right)+\frac{1}{m_{k} n_{k}}\left(\boldsymbol{F}_{k}-\nabla \cdot \pi_{k}\right) \\
\vec{\omega}_{k}=\nabla \times \boldsymbol{v}_{k} \quad ; \quad \vec{\Omega}_{k}=\frac{q_{k} \boldsymbol{B}}{m_{k}} \quad ; \quad \boldsymbol{E}=-\nabla \Phi
\end{gathered}
$$

The operator $\nabla \times \ldots$ yields the reduced vorticity equation

$$
\frac{\partial \vec{\omega}_{k}}{\partial t}-\nabla \times\left(\boldsymbol{v}_{k} \times\left(\vec{\omega}_{k}+\vec{\Omega}_{k}\right)\right)=-\frac{1}{n_{k}^{2}} \nabla p_{k} \times \nabla n_{k}
$$

where we have neglected the source terms and the dissipative terms. These equations describe also the Rossby waves if one identifies $\vec{\Omega}_{k}$ with the vorticity of the planet and makes 
the barotropic approximation $p_{k}=p_{k}\left(n_{k}\right)$ and $\nabla \cdot \boldsymbol{v}_{k}=0(k=1)$. In a plasma, however, the equation of continuity and compressibility are important and together with the approximation (6) the following conservation law of "potential vorticity" [11] holds

$$
n_{k} \frac{d}{d t}\left(\frac{\vec{\omega}_{k}+\vec{\Omega}_{k}}{n_{k}}\right)=\left(\left(\vec{\omega}_{k}+\vec{\Omega}_{k}\right) \cdot \nabla\right) \boldsymbol{v}_{k}-\frac{1}{n_{k}^{2}} \nabla p_{k} \times \nabla n_{k} \quad ; \quad \frac{d}{d t}=\frac{\partial}{\partial t}+\boldsymbol{v}_{k} \cdot \nabla
$$

which generalises the well-known vorticity equation of incompressible hydrodynamics. The right hand side is the origin of vorticity stretching [23], which in $2 D$ geometry does not occur. This equation is the basis of the generalised Hasegawa-Mima equation [11] describing the turbulence of resistive drift waves. The issue being addressed in the following is the spectrum of drift waves in a multi-species plasma.

\subsection{Drift waves}

In the following we focus the analysis on electrostatic drift waves in a time-independent magnetic field. Furthermore we neglect all dissipative mechanisms and the particle source terms. These will be included later on. The first step is to eliminate the electric potential employing the condition of quasi-neutrality. We return to the force balance (4) and write the pressure gradient as

$$
\frac{\nabla p_{k}}{n_{k}}=\nabla\left(T_{k} \ln \frac{n_{k}}{n_{0}}\right)+\left(1-\ln \frac{n_{k}}{n_{0}}\right) \nabla T_{k}
$$

Defining the generalized potential $P_{k}$

$$
P_{k}=T_{k} \ln \frac{n_{k}}{n_{0}}+q_{k} \Phi \Longrightarrow n_{k}=n_{0} \exp \left(\frac{P_{k}-q_{k} \Phi}{T_{k}}\right)
$$

makes the density a function of $P_{k}, T_{k}, \Phi . n_{0}$ is a $k$-independent reference density. The quasineutrality requires

$$
H\left(P_{k}, T_{k}, \Phi\right)=\sum_{k} q_{k} \exp \left(\frac{P_{k}-q_{k} \Phi}{T_{k}}\right)=0
$$

and because of

$$
\partial H / \partial \Phi=-\sum_{k} \frac{q_{k}^{2} n_{k}}{T_{k}}<0 \forall P_{k} \text { and } T_{k}>0
$$

this non-linear equation has always a unique solution $\Phi=\Phi\left(P_{i}, T_{i}\right), i=1,2 \ldots . K$. In a two-fluid model this inversion can easily be done analytically. Eliminating the electric potential this way makes the density a function of $P_{i}, T_{i}$ and the time derivative of the density is

$$
\frac{\partial n_{k}}{\partial t}=\frac{n_{k}}{T_{k}}\left(\frac{\partial P_{k}}{\partial t}-q_{k} \sum_{i} \frac{\partial \Phi}{\partial P_{i}} \frac{\partial P_{i}}{\partial t}\right)
$$

From Eq. (9) and (11) we get

$$
\frac{\partial \Phi}{\partial P_{i}}=\frac{1}{M} \frac{q_{i} n_{i}}{T_{i}} \quad ; \quad M=\sum_{k} \frac{q_{k}^{2} n_{k}}{T_{k}} \quad A_{i k}:=\frac{n_{k}}{T_{k}}\left(\delta_{i k}-\frac{q_{k} q_{i} n_{i}}{T_{i} M}\right)
$$

and define the symmetric matrix $A_{i k}$. This matrix is singular; the vector $\boldsymbol{z}=\left\{q_{i}\right\}$ solves the homogeneous equation $A \boldsymbol{z}=0$. However, the matrix is positive semi-definite which follows from

$$
\sum_{i k} A_{i k} \delta P_{i} \delta P_{k}=\sum_{k} \frac{T_{k}}{n_{k}}\left(\delta n_{k}\right)^{2} \geq 0
$$


In terms of the generalized potential the equations of continuity are

$$
\sum_{i} A_{i k} \frac{\partial P_{i}}{\partial t}+\nabla \cdot n_{k}\left(P_{1}, \ldots P_{K}\right) \boldsymbol{v}_{k}=0
$$

This equation advances the generalised potentials in time if the orthogonality condition is satisfied. This is nothing else but $\nabla \cdot \sum_{k} q_{k} n_{k} \boldsymbol{v}_{k}=0$. After eliminating the electric potential the reduced force balance equation is

$$
\frac{\partial \boldsymbol{v}_{k}}{\partial t}+\frac{1}{2} \nabla \boldsymbol{v}_{k}^{2}-\boldsymbol{v}_{k} \times\left(\vec{\omega}_{k}+\vec{\Omega}_{k}\right)=-\frac{1}{m_{k}}\left(\nabla P_{k}+\left(1-\ln \frac{n_{k}}{n_{0}}\right) \nabla T_{k}\right)
$$

The temperature $T_{i}(\boldsymbol{x})$ is a time-independent given function. A basic question is now: Is this system a well-posed initial value problem? Do the equations of continuity advance the generalized potential in time? Because of $\nabla \cdot \sum_{k} q_{k} n_{k} \boldsymbol{v}_{k}=0$ the orthogonality condition is satisfied and the system Eq. (15) inverted with respect to the time derivatives of $P_{k}$. Given the generalised potentials, the force balance advances the velocity in time.

In order to demonstrate the drift wave character we consider in isothermal plasma $\nabla T_{k}=0$ and linearise the equations around $\boldsymbol{v}_{k}^{0}=0$ (no zeroth order flow). Exponential ansatz in $t$ yields

$$
i \omega \delta \boldsymbol{v}_{k}-\delta \boldsymbol{v}_{k} \times \vec{\Omega}_{k}=-\frac{1}{m_{k}} \nabla \delta P_{k} \quad \text { and } \quad i \omega \sum_{i} A_{i k} \delta P_{i}+\delta \boldsymbol{v}_{k} \cdot \nabla N_{k}+N_{k} \nabla \cdot \delta \boldsymbol{v}_{k}=0
$$

$N_{k}$ is the unperturbed density. Eliminating $\delta \boldsymbol{v}_{k}$ from the first equation and inserting the result into the second equation yields the dispersion relation for parallel sound waves and perpendicular drift waves.

$$
\begin{gathered}
\delta v_{k, \|}=-\frac{1}{i \omega} \frac{\vec{\Omega}_{k}}{m_{k} \Omega_{k}^{2}} \vec{\Omega}_{k} \cdot \nabla \delta P_{k} \quad ; \quad \delta \boldsymbol{v}_{k, \perp} \approx \frac{\vec{\Omega}_{k} \times \nabla \delta P_{k}}{m_{k} \Omega_{k}^{2}}-i \omega \frac{\nabla \perp \delta P_{k}}{m_{k} \Omega_{k}^{2}} \\
i \omega \sum_{i} A_{i k} \delta P_{i}-i \omega N_{k} \nabla \cdot \frac{\nabla_{\perp} \delta P_{k}}{m_{k} \Omega_{k}^{2}}+\left(\frac{\vec{\Omega}_{k} \times \nabla \delta P_{k}}{m_{k} \Omega_{k}^{2}}\right) \cdot \nabla N_{k}-N_{k} \nabla \cdot\left(\frac{1}{i \omega} \frac{\vec{\Omega}_{k}}{m_{k} \Omega_{k}^{2}} \vec{\Omega}_{k} \cdot \nabla \delta P_{k}\right)=0
\end{gathered}
$$

In slab geometry and homogeneous magnetic field, this eigenvalue problem can be converted into an algebraic equation. The last term describes the parallel sound waves. Setting $k_{\|}=0$ yields the dispersion relation of drift waves

$$
i \omega\left(\sum_{i} A_{i k} \delta P_{i}-N_{k} \nabla \cdot \frac{\nabla_{\perp} \delta P_{k}}{m_{k} \Omega_{k}^{2}}\right)+\left(\frac{\vec{\Omega}_{k} \times \nabla \delta P_{k}}{m_{k} \Omega_{k}^{2}}\right) \cdot \nabla N_{k}=0
$$

In general this eigenvalue problem has as many solutions $\omega_{i}, i=1, \ldots K$ as particle species. The matrix $A_{i k}$ couples the drift waves of the various particles species; without the coupling term $A_{i k} \longrightarrow n_{k} / T_{k} \delta_{i k}$ the dispersion relation decouples into a system of independent drift waves for every particle species. However, setting $k_{\|}=0$ is not correct since the motion parallel to the magnetic field is not treated properly. The parallel force balance is

$$
i \omega \vec{\Omega}_{k} \cdot \delta \boldsymbol{v}_{k}=-\frac{1}{m_{k}} \vec{\Omega}_{k} \cdot \nabla \delta P_{k}
$$

Setting the determinant of the homogeneous system equal to zero yields the desired dispersion relation. This determinant is a polynomial of order $2 K$. In a two-component plasma this is a fourth-order polynomial which describes two slow ion waves (drift wave and sound wave) and two fast electron waves. Often the approximation $m_{e} \longrightarrow 0$ is made which brings the electron sound velocity to infinity and $\delta P_{e} \longrightarrow 0$. Only ion sound and drift waves are left in this case. 
This dispersion relation does not describe the growth rate of the drift waves; for this purpose the friction forces must be retained in the parallel motion. Strictly speaking the analysis is only valid for density-gradient-driven drift waves, however including the temperature gradient and the energy equation would extend the investigation also to ITG-modes in a multi-species plasma.

In summary, we have shown that in plasmas with several ion species also more than one drift wave can exist. The coupling between the various ion drift waves is provided by the non-diagonal terms in the matrix $A_{i k}$. In particular, this can be of interest in a $D-T$-mixture or $H-D$ mixture. Also heavy ion impurities may play a role since the the coupling to the main plasma goes with density and charge of the impurities. The dominating role of the electric potential in a two-component plasma is replaced by the generalised potential of each particle species. The non-linear behaviour of these drift waves and the generalisation of the Hasegawa-Mima equation and Hasegawa-Wakatani equations will be investigated in a separate paper.

\subsection{Spin-up equations}

To proceed further we return to the momentum balance Eq. (1) and convert this into a dimensionless form. We introduce a reference magnetic field $B_{0}$, a reference density $n_{0}$ and a temperature $T_{0}$. The length scale is $a$. The plasma pressure is replaced by the plasma beta $\beta_{k}=\mu_{0} p_{k} / B_{0}^{2}$. Furthermore, we make use of the plasma frequency $\omega_{p}$ and the classical skin $\operatorname{depth} \delta_{e}$

$$
\omega_{p}=\frac{e^{2} n_{0}}{m_{e}} \quad \text { and } \quad \delta_{e}=\frac{c}{\omega_{p}}
$$

We introduce the non-dimensional velocity $\boldsymbol{u}$ and the non-dimensional electric field $\boldsymbol{E}$ by

$$
\boldsymbol{u}:=\frac{a \boldsymbol{v}}{\delta_{e}^{2} \Omega_{e}} \quad ; \quad \boldsymbol{E}:=\frac{a \boldsymbol{E}}{\delta_{e}^{2} B_{0} \Omega_{e}} \quad ; \quad \boldsymbol{b}=\frac{\boldsymbol{B}}{B_{0}} \quad ; \quad \Omega_{e}=\frac{e B_{0}}{m_{e}} \quad ; \quad d \tau=\Omega_{e} d t
$$

$m_{e}$ is the electron mass and $e$ the charge of electrons. $\tau$ is the normallzed time scale. In the following $\boldsymbol{E}$ is the non-dimensional electric field. The collision interaction between charged particles is characterized by the non-dimensional collision frequency

$$
f_{i k}=\frac{l_{11}^{i k}}{m_{e} n_{0} \nu_{e e}}
$$

$\nu_{e e}\left(T_{0}, n_{0}\right)$ is the electron collision frequency at the reference point $n_{0}, T_{0}$. Using these definitions the momentum balance and the equation of continuity in non-dimensional formulation become

$$
\frac{m_{k}}{m_{e}} \frac{\partial n_{k} \boldsymbol{u}_{k}}{\partial \tau}+\nabla \cdot\left(\frac{\mu_{0} \pi_{k}}{a B_{0}^{2}}+\frac{m_{k}}{m_{e}} \frac{\delta_{e}^{2}}{a^{2}} n_{k} \boldsymbol{u}_{k}: \boldsymbol{u}_{k}\right)=-\nabla \beta_{k}+\sigma_{k} n_{k}\left\{\boldsymbol{E}+\boldsymbol{u}_{k} \times \boldsymbol{b}\right\}+\sum_{i} \frac{\nu_{e e}}{\Omega_{e}} f_{i k} \boldsymbol{u}_{i}
$$

and

$$
\frac{\partial n_{k}}{\partial \tau}+\frac{\delta_{e}^{2}}{a^{2}} \nabla \cdot n_{k} \boldsymbol{u}_{k}=\frac{S_{k}}{n_{0} \Omega_{e}}:=s_{k}
$$

$n_{k}$ is the normalized density and $\sigma_{k}$ is the charge of the particles diveded by the electron charge $e$. This equation is a convenient tool in order to estimate the importance of the various terms. Since the skin depth $\delta_{e}$ is a small value the inertial forces are rather small compared with the other terms. However, this is only true in a direction normal to magnetic surfaces, where pressure gradient, electric field and the $\boldsymbol{u} \times \boldsymbol{b}$ - term are the dominating ones. In the tangential direction these forces are zero or very small and inertial forces, viscous forces and friction forces must be taken into account. The source terms describe the ionisation and recombination processes. In a multi-component fusion plasma the source terms also describe the nuclear processes. All source 
terms are continuous functions of plasma densities, neutral density and temperature. Since all these processes conserve the electric charge, i.e. $\sum_{k} q_{k} S_{k}=0$, the particle fluxes are ambipolar in steady-state conditions.

After introducing a coordinate system $V, \theta, \varphi$ on the magnetic surface and the associated contravariant base vectors $\boldsymbol{e}_{V}, \boldsymbol{e}_{p}, \boldsymbol{e}_{t}$ we decompose the force balance with the aid of these base vectors. Here, $p$ and $t$ denote the poloidal and the toroidal base vector. $V$ is the surface label and $\boldsymbol{e}_{V}$ is the normal base vector. A special choice is when we set $V$ equal to the volume of the magnetic surface. All contravariant base vectors are incompressible by definition. For the moment there is no need to specify the coordinate system in detail. The normal ( or radial) force balance can be approximated by

$$
0=-\boldsymbol{e}_{V} \cdot \nabla \beta_{k}+\sigma_{k} n_{k} \boldsymbol{e}_{V} \cdot\left(\boldsymbol{E}+\boldsymbol{u}_{k} \times \boldsymbol{b}\right)
$$

which assumes that radial inertial forces, viscous forces and friction forces are small compared with the pressure gradient, radial electric field and Lorentz forces. Summing up these equations over the particle species yields

$$
0=-\boldsymbol{e}_{V} \cdot \nabla \beta+\boldsymbol{e}_{V} \cdot(\boldsymbol{j} \times \boldsymbol{b}) \quad ; \quad \boldsymbol{j}=\sum_{k} \sigma_{k} n_{k} \boldsymbol{u}_{k} \quad ; \quad \beta=\sum_{k} \beta_{k}
$$

which is the well-known force balance of the ideal plasma equilibrium. Let $\boldsymbol{t}$ be a tangential base vector on the magnetic surface - either $\boldsymbol{e}_{p}$ or $\boldsymbol{e}_{t}$ or a linear combination of both. The tangential force balance is

$$
\begin{aligned}
\frac{m_{k}}{m_{e}} \frac{\partial \boldsymbol{t} \cdot n_{k} \boldsymbol{u}_{k}}{\partial \tau} & +\boldsymbol{t} \cdot \nabla \cdot\left\{\frac{\mu_{0} \pi_{k}}{B_{0}^{2}}+\frac{m_{k}}{m_{e}} \frac{\delta_{e}^{2}}{a^{2}} n_{k} \boldsymbol{u}_{k}: \boldsymbol{u}_{k}\right\}=-\boldsymbol{t} \cdot \nabla \beta_{k}+\sigma_{k} n_{k} \boldsymbol{t} \cdot \boldsymbol{E} \\
& +\sigma_{k} n_{k} \boldsymbol{u}_{k} \cdot(\boldsymbol{b} \times \boldsymbol{t})+\sum_{i} \frac{\nu_{e e}}{\Omega_{e}} f_{i k} \boldsymbol{t} \cdot \boldsymbol{u}_{k}
\end{aligned}
$$

which is also the evolution equation of the tangential momenta. The tangential force balance shows that one of the driving terms of the tangential flow is the $\sigma_{k} n_{k} \boldsymbol{u} \cdot(\boldsymbol{b} \times \boldsymbol{t})$-term. In terms of the base vectors the magnetic field is $\boldsymbol{b}=b^{t} \boldsymbol{e}_{t}+b^{p} \boldsymbol{e}_{p}$. The vector product $\boldsymbol{e}_{p} \times \boldsymbol{e}_{t}=J \nabla V$ is proportional to the normal vector of the magnetic surface; $J$ is the Jacobian of the coordinate system, The vector $(\boldsymbol{b} \times \boldsymbol{t}) \propto \nabla V$ points in the normal direction which shows that the vector $n_{k} \boldsymbol{u} \cdot(\boldsymbol{b} \times \boldsymbol{t}) \propto n_{k} \boldsymbol{u} \cdot \nabla V$ is proportional to the local particle flux across the magnetic surface. The proportionality factor depends on the choice of the coordinate system. In magnetic cordinates (or straight field line coordinates) the components $b^{p}(V), b^{t}(V)$ are surface functions and the proportionality factor is the Jacobian of the coordinate system. The Hamada coordinate system has a Jacobian $J=1$ and and in this case the term $n_{k} \boldsymbol{u} \cdot(\boldsymbol{b} \times \boldsymbol{t})$ is equal to the local radial particle flux times $b^{t}$ or $b^{p}$ depending on the choice of the tangential base vector. How to compute the Hamada coordinate system in tokamak geometry has been demonstrated in [24],[27]. Further driving terms are the tangential inertial forces and the tangential electric field. Viscosity and friction slow down the tangential flow. The force $\boldsymbol{t} \cdot \nabla \beta_{k}$ is zero when we average the force over the magnetic surface. The averaging procedure is defined by

$$
<g>=\int \frac{g d f}{|\nabla V|} \quad ; \quad<1>=1
$$

and it can be easily shown that $<\boldsymbol{e}_{p} \cdot \nabla \beta_{k}>=<\boldsymbol{e}_{t} \cdot \nabla \beta_{k}>=0$. This is valid for any single-valued scalar $g$ instead of $\beta_{k}$; $d f$ is the surface element of the magnetic surface. Applying this averaging procedure to the tangential force balance yields

$$
\begin{aligned}
\frac{m_{k}}{m_{e}} \frac{\partial<\boldsymbol{t} \cdot n_{k} \boldsymbol{u}_{k}>}{\partial \tau} & +<\boldsymbol{t} \cdot \nabla \cdot\left\{\frac{\mu_{0} \pi_{k}}{B_{0}^{2}}+\frac{m_{k}}{m_{e}} \frac{\delta_{e}^{2}}{a^{2}} n_{k} \boldsymbol{u}_{k}: \boldsymbol{u}_{k}\right\}>=\sigma_{k}<n_{k} \boldsymbol{t} \cdot \boldsymbol{E}> \\
& +\sigma_{k}<n_{k} \boldsymbol{u}_{k} \cdot(\boldsymbol{b} \times \boldsymbol{t})>+\sum_{i} \frac{\nu_{e e}}{\Omega_{e}} f_{i k}<\boldsymbol{t} \cdot \boldsymbol{u}_{k}>
\end{aligned}
$$


The friction coefficients $f_{i k}$ are treated as surface functions. Using a straight field lines system we find

$$
\begin{aligned}
& <n_{k} \boldsymbol{u}_{k} \cdot\left(\boldsymbol{b} \times \boldsymbol{e}_{t}\right)>=b^{p}(V) \int J n_{k} \boldsymbol{u}_{k} \cdot d \boldsymbol{f} \\
& <n_{k} \boldsymbol{u}_{k} \cdot\left(\boldsymbol{b} \times \boldsymbol{e}_{p}\right)>=-b^{t}(V) \int J n_{k} \boldsymbol{u}_{k} \cdot d \boldsymbol{f}
\end{aligned}
$$

In the Hamada system the Jacobian is unity and the right hand side is proportional to the integrated particle flux across the magnetic surface. The integrated particle flux thus contributes to the driving terms of the tangential particle fluxes, however the sum over all particle species is zero because of the ambipolarity of the radial particle fluxes. Quasi-neutrality of the plasma (also in the turbulent state) makes the sum of all tangential electric forces zero $\left.\sum_{k} \sigma_{k}<n_{k} \boldsymbol{t} \cdot \boldsymbol{E}\right\rangle=0$. The same result holds for the sum of the friction forces (even if we retain the heat fluxes in $\boldsymbol{F}_{k}$. Employing the Hamada coordinate system $(J=1)$ and summing up all surface-averaged momentum equations we find

$$
\frac{\partial}{\partial \tau} \sum_{k} \frac{m_{k}}{m_{e}}<\boldsymbol{t} \cdot n_{k} \boldsymbol{u}_{k}>+\sum_{k}<\boldsymbol{t} \cdot \nabla \cdot\left\{\frac{\mu_{0} \pi_{k}}{B_{0}^{2}}+\frac{m_{k}}{m_{e}} \frac{\delta_{e}^{2}}{a^{2}} n_{k} \boldsymbol{u}_{k}: \boldsymbol{u}_{k}\right\}>=0
$$

The time evolution of the total surface averaged momentum is not affected by the friction forces, the averaged Lorentz forces and the electric field, while these have a significant effect on the spin-up of the separate particle species.

Spin-up of poloidal and toroidal rotation in tokamaks has attracted the attention of many authors since T. Stringer [6] published the first paper on this issue.

\subsection{Viscosity}

The viscous tensor depends on the regime of collisionality; in a collisional plasma we may utilize the Braginskii viscosity [17] while in the long-mean-free-path regime the neoclassical viscosity results from a kinetic equation. The Braginskii approximation holds in collisional plasmas and is written as

$$
\pi_{i k}=-\sum_{0}^{4} \eta_{l} W_{l, i k} \quad ; \quad W_{l, i k}=\sum_{\mu \nu} A_{l, i k}^{\mu \nu} W_{\mu \nu}
$$

The tensors $\mathbf{A}_{l}$ depend on the magnetic field and their coefficients are of the order unity. $\eta_{l}$ are the viscosity coefficients and $W_{\mu \nu}$ is the rate of strain tensor

$$
W_{\mu \nu}=\left(\frac{\partial v_{\mu}}{\partial x_{\nu}}+\frac{\partial v_{\nu}}{\partial x_{\mu}}-\frac{2}{3} \nabla \cdot \boldsymbol{v} \delta_{\mu \nu}\right) \text { or } \mathbf{W}[\boldsymbol{v}]=\nabla: \boldsymbol{v}+(\nabla: \boldsymbol{v})^{T}-\frac{2}{3} \nabla \cdot \boldsymbol{v}
$$

$\eta_{0, k}=a_{0} p_{k} \tau_{k}, a_{0} \approx 1$ is the largest of the viscous coefficients; $p_{k}$ is the pressure of the particle species and $\tau_{k}$ is the like-particle collision time. In the limit of strong magnetic fields the coefficients of shear viscosity $\eta_{1, k}$ and $\eta_{2, k}$ are smaller by a factor $\approx x^{-2}$, with $x=\Omega_{k} \tau_{k} \gg 1$. The coefficients of gyro-viscosity $\eta_{3, k}$ and $\eta_{4, k}$ are approximately equal to $\eta_{0} x^{-1}$. $\Omega_{k}$ is the gyrofrequency. In the limit of weak magnetic fields we get $\eta_{0, k}=\eta_{1, k}$. The non-dimensional viscous coefficients are

$$
\eta_{0, k}=\frac{m_{k}}{m_{e}} \frac{v_{t h, k}^{2} \tau_{k}}{\Omega_{e} a^{2}} n_{k} \quad, \quad \eta_{1, k}=\eta_{0, k}\left(\Omega_{k} \tau_{k}\right)^{-2} \quad ; \quad \eta_{2, k}=\eta_{0, k}\left(\Omega_{k} \tau_{k}\right)^{-1}
$$

Here $n_{k}$ is the non-dimensional density. In order to derive this form we have used $p_{k}=n_{k} T_{k}$ and the thermal velocity defined by $v_{t h, k}^{2}=T_{k} / m_{k} \cdot \eta_{0, k}$ describes the magnetic pumping effect, 
which depends on the inhomogeneity of the magnetic field. The shear viscosity $\eta_{1, k}$ is the relevant transport coefficient for radial momentum transport written in non-dimensional form

$$
\eta_{1, k}=\frac{m_{k}}{m_{e}} \frac{\rho_{k}^{2} \nu_{k}}{\Omega_{e} a^{2}} n_{k}
$$

$\nu_{k}$ is the like-particle collision frequency. The gyro radius is the characteristic radial length of momentum transport and $\nu_{k}$ the characteristic frequency. Although the gyro viscosity is larger than the shear viscosity it is not dissipative and has not the same effect as the shear viscosity. The bulk viscosity $\eta_{0, k} W_{0, i k}$ (also called parallel viscosity since it depends on $p_{\|}-p_{\perp}$ ) is the origin of the magnetic pumping effect which dominates in toroidal geometry. In tokamaks, magnetic pumping does not affect the toroidal direction, only the the poloidal flow is damped. The full Braginskii viscosity has been analysed by Stacey and Sigmar for tokamak geometry [18] identifying the role of the gyro viscosity in axisymmetric geometry.

Magnetic pumping in stellarators slows down plasma flow in all directions, the details depend on the structure of mod $B$ on the magnetic surface [27]. As already mentioned, the viscosity should be supplemented by an additional term containing the heat flux [21][19]. We do not claim that this term may be neglected, however, in view of the experimental result in $\mathrm{W} 7-\mathrm{AS}$, ignoring this term atr the moment may be justified a posteriori. If we consider the heat flux vector $\boldsymbol{q}$ as given it adds just another inhomogeneous term to the momentum balance as the heat-flux terms in the friction forces do. These terms provide a link to the energy balance and certainly play a role in determining a power threshold for the onset of the H-mode. However, the HDH-mode in W 7-AS is triggered by a gas puff at fixed NBI-heating suggesting that the phenomenon may be understood on the basis of particle and momentum balance alone.

The power dissipated by viscous forces slows down any plasma motion. In order to demonstrate this we compute the integral over the plasma domain $\Omega$.

$$
P=\int_{\Omega} \boldsymbol{v} \cdot \nabla \cdot \pi d^{3} \boldsymbol{x}=\sum_{l} \int_{\Omega} \boldsymbol{v} \cdot \nabla \cdot \eta_{l} \mathbf{A}_{l} \mathbf{W} d^{3} \boldsymbol{x}
$$

By partial integration we get

$$
P=-\frac{1}{2} \sum_{l} \int_{\Omega} \eta_{l} \mathbf{W} \mathbf{A}_{l} \mathbf{W} d^{3} \boldsymbol{x}+\int_{\dot{\Omega}} \boldsymbol{v} \cdot \pi \cdot d \boldsymbol{f}
$$

Since the viscous tensor is symmetric and has zero trace the tensor $\nabla: \boldsymbol{v}$ can be replaced by $\mathbf{W}$ in the volume integral on the right hand side. The surface integral vanishes with homogeneous boundary conditions $(\boldsymbol{v}=0$ on the boundary). The details of the volume integral have been investigated in [22]. It turns out that the tensors $\mathbf{A}_{3}, \mathbf{A}_{4}$ representing the gyro viscosity are antisymmetric leading to $\mathbf{W} \mathbf{A}_{l} \mathbf{W}=0, l=3,4$. The gyro viscosity does not provide any contribution to the power dissipated by the Braginskii viscosity; this is done by the bulk viscosity and the shear viscosity. When discussing the entropy production rate, Braginskii has mentioned this result without detailed proof. Furthermore, it can be shown that $P$ is always negative. Braginskii gives a simple form of the matrices $\mathbf{A}_{l} \mathbf{W}$ for the case of a homogeneous magnetic field; here the properties mentioned above can be easily verified.

Standard neoclassical theory provides us with the equivalent of the bulk viscosity and basically describes the magnetic pumping. However, neoclassical viscosity - as a result of the kinetic equation - depends on the radial electric field. We shall not go into this further, since the main interest of this paper is focussed on a simple geometry where neoclassical effects are unimportant and shear viscosity and gyro viscosity are the dominating ones.

Viscous damping in non-axisymmetric equilibria - in particular in view of stellarator configurations - has been investigated by Wobig and Kisslinger [27]. The magnetic pumping effect 
is the dominating one, which slows down poloidal and toroidal rotation. The matrix of viscous coefficients in general toroidal configurations is

$$
\eta_{0, k}\left(\begin{array}{cc}
<\left(\boldsymbol{e}_{p} \cdot \frac{\nabla B}{B}\right)^{2}> & <\left(\boldsymbol{e}_{p} \cdot \frac{\nabla B}{B}\right)\left(\boldsymbol{e}_{t} \cdot \frac{\nabla B}{B}\right)> \\
<\left(\boldsymbol{e}_{t} \cdot \frac{\nabla B}{B}\right)\left(\boldsymbol{e}_{p} \cdot \frac{\nabla B}{B}\right)> & <\left(\boldsymbol{e}_{t} \cdot \frac{\nabla B}{B}\right)^{2}>
\end{array}\right)
$$

which includes the tokamak case $\boldsymbol{e}_{t} \cdot \nabla B=0$. In stellarators, magnetic pumping provides a coupling beween poloidal and toroidal flow. Optimising stellarator configurations [25][26] implies a minimisation of $\delta|B|$ on magnetic surfaces so that at least in one of the coordinate lines the variation of $|B|$ is zero or negligibly small. The implications of $\boldsymbol{e}_{p} \cdot \nabla B \longrightarrow 0$ are far-reaching: zero geodesic curvature of field lines, zero Pfirsch-Schlüter currents, no radial drift of particles and no neoclassical transport [27][28].

\subsection{Reynolds averaged equations}

In order to deal with turbulent effects we start from the Reynolds averaged momentum balance equations (RANS-equation). Reynolds averaging means to average over the longest time scale inherent in the turbulent plasma, thus averaging over the fast time scale of drift waves and the slower time scale which is characteristic for the evolution of zonal flow. Let $D:=$ $[\tau-T \leq t \leq \tau+T]$ be a domain in $t$ and $F(t-\tau)$ a differentiable filter function which is zero outside of this domain and positive inside. The properties are

$$
F>0 \text { if } t \in D \quad ; \quad F=0 \text { if } t \ni D \quad ; \quad \int_{\infty}^{\infty} F d t=1
$$

Averaging a function $g(t)$ over $D$ leads to

$$
\bar{g}=\int_{\infty}^{\infty} F(t-\tau) g(t) d t \quad ; \quad \int_{\infty}^{\infty} F(t-\tau) \frac{g(t)}{d t} d t=\frac{d \bar{g}}{d \tau}
$$

By proper choice of the width $T$ one can average over a short or a long time scale. The velocities are replaced by $\overline{\boldsymbol{u}}+\delta \boldsymbol{u}$ and the densities by $\bar{n}+\delta n$. The electric field is $\overline{\boldsymbol{E}}+\delta \boldsymbol{E}$. The first term in these quantities is the time-averaged value and the $\delta$-term describes the fluctuating part. Averaging the momentum balance over the turbulent time scale provides us with the equivalent to the Reynolds equations of hydrodynamincs [23]. Turbulence gives rise to additional forces in the momentum balance. The Reynolds averaged equations are

$$
\nabla \cdot\left\{\frac{\mu_{0} \pi_{k}}{B_{0}^{2}}+\frac{m_{k}}{m_{e}} \frac{\delta_{e}^{2}}{a^{2}} \overline{n_{k} \boldsymbol{u}_{k}: \boldsymbol{u}_{k}}\right\}=-\nabla \bar{\beta}_{k}+\sigma_{k} \bar{n}_{k} \overline{\boldsymbol{E}}+\sigma_{k} \overline{\delta n_{k} \delta \boldsymbol{E}}+\sigma_{k} \overline{n_{k} \boldsymbol{u}_{k}} \times \boldsymbol{b}+\overline{\boldsymbol{F}}_{k}
$$

$k=1, \ldots N$. The term $\overline{m_{k} \delta \boldsymbol{u}_{k}: \delta \boldsymbol{u}_{k}}$ is known as Reynolds stress in hydrodynamics. However, this is not the only contribution of the inertial forces; these are

$$
\begin{aligned}
\overline{n_{k} \boldsymbol{u}_{k}: \boldsymbol{u}_{k}} & =\overline{\boldsymbol{u}}_{k}: \overline{n_{k} \boldsymbol{u}_{k}}+\overline{\delta \boldsymbol{u}_{k}: \delta\left(n_{k} \boldsymbol{u}_{k}\right)} \\
& =\bar{n}_{k} \boldsymbol{u}_{k}: \overline{\boldsymbol{u}}_{k}+\overline{\delta\left(n_{k} \boldsymbol{u}_{k}\right): \delta \boldsymbol{u}_{k}} \\
& =\bar{n}_{k} \overline{\boldsymbol{u}}_{k}: \overline{\boldsymbol{u}}_{k}+\bar{n}_{k} \overline{\delta \boldsymbol{u}_{k}: \delta \boldsymbol{u}_{k}}+\overline{\delta n_{k} \delta\left(\boldsymbol{u}_{k}: \boldsymbol{u}_{k}\right)}
\end{aligned}
$$

The friction coefficients in the momentum balance are assumed to be time independent. The viscous tensor is a linear functional of the time-averaged distribution function. Large efforts have been made in hydrodynamics to close the system by relating the turbulent forces to the mean flow. This has led to the concept of eddy viscosity which describes the enhanced momentum 
transport by the small eddies [29]. The turbulent force by electric field fluctuations has no counterpart in hydrodynamics and many efforts have been made in plasma physics to start from gradient driven instabilities and to model the turbulent terms by an anomalous transport coefficient multiplying the gradients. The equation of continuity becomes

$$
\nabla \cdot \overline{n_{k} \boldsymbol{u}_{k}}=\bar{s}_{k} \quad ; \quad \sum_{k} q_{k} \bar{s}_{k}=0 \quad \Longrightarrow \sum_{k} q_{k} \int_{S} \overline{n_{k} \boldsymbol{u}_{k}} \cdot d \boldsymbol{f}=0
$$

The source terms on the right hand side describe the ionisation and recombination processes and since these processes conserve the electric charge the particle fluxes are intrinsically ambipolar. In summary, the radial force balance is approximated by

$$
0=-\boldsymbol{n} \cdot \nabla \bar{\beta}_{k}+\sigma_{k} \overline{n_{k} \boldsymbol{e}_{V} \cdot \boldsymbol{E}}+\sigma_{k}{\overline{n_{k}}}_{k} \cdot\left(\boldsymbol{b} \times \boldsymbol{e}_{V}\right)
$$

and the tangential force balance by

$$
\begin{aligned}
& <\boldsymbol{t} \cdot \nabla \cdot\left(\frac{\mu_{0} \pi_{k}}{B_{0}^{2}}+\frac{m_{k}}{m_{e}} \frac{\delta_{e}^{2}}{a^{2}} \overline{n_{k} \boldsymbol{u}_{k}: \boldsymbol{u}_{k}}\right)> \\
& =\sigma_{k}<\overline{n_{k} \boldsymbol{t} \cdot \boldsymbol{E}}>+\sigma_{k}<\overline{n_{k} \boldsymbol{u}} \cdot(\boldsymbol{b} \times \boldsymbol{t})>+\frac{\nu_{e}}{\Omega_{e}} \sum_{i} f_{i k}<\boldsymbol{t} \cdot \overline{\boldsymbol{u}}_{k}>
\end{aligned}
$$

The relationship to cyclonic motion in the earth's atmosphere can be seen by dropping the friction forces and the electric fields and retaining one fluid only. The magnetic field is replaced by the $\vec{\Omega}$ - component - or Coriolis parameter - in the tangential plane ( $\beta$-plane). $\vec{\Omega}$ is the vorticity of the planet. There, the radial balance (48) is called geostrophic equilibrium while the circulation is governed by inertial forces, viscous forces and the spin-up force due to the radial flux times the Coriolis parameter [7]. It is the radial influx together with the Coriolis parameter which spins up hurricanes and cyclones. In the review article of Terry [30] the relation to atmospheric phenomena has been discussed in more detail. In the following we will focus the attention on a cylindrical plasma.

\section{Plasma cylinder}

Cylindrical geometry is often used as a model for toroidal plasmas, being aware that such effects as poloidal asymmetries, geodesic curvature, etc., do not occur in cylinder geometry. However, turbulence, anomalous transport and bifurcations arising from non-linearities also exist in cylinder geometry. In cylinder geometry $(r, \theta, z)$ the magnetic field has a $z$-component $B_{z}$ and a poloidal component $B_{\theta}(r)$. Both are functions of the radial coordinate $r$. The plasma is confined by diamagnetic currents flowing in a domain $\Omega$ which is limited by the radii $r=r_{0}$ and $r=1 . r$ is the non-dimensional radius of the plasma cylinder. In a cylindrical coordinate system $r, \theta, z$ the Jacobian is proportional to $1 / r$. Instead of $r$ we introduce $V=r^{2} / 2$ (volume per length) and define the base vectors

$$
\boldsymbol{e}_{p}=\nabla V \times \nabla z ; \quad \boldsymbol{e}_{t}=\nabla \theta \times \nabla V ; \quad \boldsymbol{e}_{V}=\nabla z \times \nabla \theta
$$

which is a rectangular system with a Jacobian equal to unity. Furthermore we find $\boldsymbol{e}_{p} \cdot \nabla \theta=$ $1 ; \boldsymbol{e}_{t} \cdot \nabla z=1 ; \boldsymbol{e}_{p} \cdot \nabla V=1, d V=r d r,|\nabla V|^{2}=2 V$. The magnetic field in this system is $\boldsymbol{b}=b^{z}(V) \boldsymbol{e}_{t}+b^{p}(V) \boldsymbol{e}_{p}$. The poloidal field $b_{p}(V)$ is proportional to the axial current. All mean plasma parameters are functions of the radial coordinate alone $\bar{n}_{k}(V), \bar{T}_{k}(V), \bar{\beta}_{k}(V), \Phi(V)$. $\Phi(V)$ is the surface averaged electric potential. The contravariant representation of mean plasma velocity is $\overline{\boldsymbol{u}}=u^{t}(V) \boldsymbol{e}_{t}+u^{p}(V) \boldsymbol{e}_{p}+u^{V}(V) \boldsymbol{e}_{V}$. The first two components describe the plasma 
flow on the magnetic surface, the last component carries the radial plasma loss. The velocity shear of the tangential flow is the derivative with respect to $V$

$$
\boldsymbol{g}=\frac{d u^{t}(V)}{d V} \boldsymbol{e}_{t}+\frac{d u^{p}(V)}{d V} \boldsymbol{e}_{p}
$$

If $u^{p}=$ const. the poloidal flow is a rigid rotation; $u^{p}$ is the angular velocity of poloidal rotation. The covariant components of the velocity are

$$
u_{p}=u^{p}|\nabla V|^{2}=u^{p} 2 V \quad ; \quad u_{V}=\frac{u^{V}}{2 V} \quad ; \quad u_{t}=u^{t}
$$

The mean electric field has no poloidal component, however in tokamaks there is an axial component due to the loop voltage while this is absent in stellarators. Next, we analyse the right hand side of eq. (49) in the cylinder.

$$
\begin{aligned}
& \sigma_{k}<\overline{\delta n_{k} \boldsymbol{e}_{p} \cdot \delta \boldsymbol{E}}>+\sigma_{k} b_{z}(V) F_{k}+<\boldsymbol{e}_{p} \cdot \boldsymbol{e}_{p}>\frac{\nu_{e}}{\Omega_{e}} \sum_{i} f_{i k} u_{k}^{p} \\
& \sigma_{k}<\overline{\delta n_{k} \boldsymbol{e}_{t} \cdot \delta \boldsymbol{E}}>-\sigma_{k} b_{p}(V) F_{k}+<\boldsymbol{e}_{t} \cdot \boldsymbol{e}_{t}>\frac{\nu_{e}}{\Omega_{e}} \sum_{i} f_{i k} u_{k}^{t}+\sigma_{k}<\bar{n}_{k} \boldsymbol{e}_{t} \cdot \overline{\boldsymbol{E}}>
\end{aligned}
$$

The last term is the effect of the mean axial electric field (toroidal loop voltage). $F_{k}$ is the radial integrated particle flux. The particle flux $F_{k}$ is equal to the integrated source terms (integrated between $0 \leq x \leq V$. The source of hydrogen ions is the neutral gas and thus hydrogen ion flux is restricted by the penetration length of neutral hydrogen. In a fusion plasma, $\alpha$-particles are produced mainly in the plasma center; therefore the alpha particle flux is non-zero in the central region. Since a source of $\alpha$-particles is a sink for deuterium and tritium, in the central region there is an influx of deuterium and tritium while in outer regions, depending on the penetration length of pellet refueling, there is a diffusive outflux of $D, T$. Ionisation and recombination of impurities occurs everywhere in the plasma and therefore finite impurity fluxes will be expected everywhere in the plasma. The source terms of electrons include all ionisation and recombination processes in the plasma; only in a pure hydrogen plasma are ion sources and electron sources equal. Because of the charge conservation of the atomic processes the sum of all source terms times the charge is zero which implies the inherent ambipolarity of the fluxes $F_{k}$. As shown in Eq. (53) the net radial flux produces a spin-up force both in poloidal and axial direction. In a hydrogen plasma with a small amount of impurities this spin-up force is restricted to a boundary layer determined by the penetration length of the neutrals. Experimental results in DIII-D [2] support the idea that an H-mode density barrier is correlated to the penetration depth of neutrals. In the Hinton-Staebler model [1] of an edge transport barrier, the ion source plays an important role in steepening the density gradient and the gradient of the $\boldsymbol{E} \times \boldsymbol{B}$ shear flow which reduces the turbulent transport.

If we neglect the viscous and inertial forces and set the terms in Eqs. (53) equal to zero, these relations provide us with an algebraic relation between the radial fluxes and the poloidal and axial mean velocities, the so-called flux-friction relationships introduced by Hirshman and Sigmar [16]. The $\delta \boldsymbol{E}$-terms represent the anomalous particle fluxes and the term with the axial electric field drives the axial (or toroidal) current. Obviously there are two alternative ways to express the radial flux. However, if the viscous and inertial forces are retained, the fluxfriction relationships are differential equation for the poloidal and axial (toroidal) velocities $u_{k}^{p}$ and $u_{k}^{t}$ and the radial fluxes are given by the source terms. The inertial forces include first order derivatives of the velocity and the viscous forces introduce second order derivatives.

In the following we compute the viscous forces on a tangential flow $u^{p}(V) \boldsymbol{e}_{p}+u^{t}(V) \boldsymbol{e}_{t}$. Using Gauss' theorem we can write the poloidal viscous and axial viscous force as follows

$$
<\boldsymbol{e}_{p} \cdot \nabla \cdot \pi_{k}>=\frac{d}{d V} \int \boldsymbol{e}_{p} \cdot \pi_{k} \cdot d \boldsymbol{f}-<\nabla: \boldsymbol{e}_{p} \cdot \pi_{k}>\quad ; \quad<\boldsymbol{e}_{t} \cdot \nabla \cdot \pi_{k}>=\frac{d}{d V} \int \boldsymbol{e}_{t} \cdot \pi_{k} \cdot d \boldsymbol{f}
$$


As shown in eq. (34), the viscous tensor is a linear differential operator of first order on the velocity. The rate of strain tensor is the only place where the velocity occurs. Applying the operator $\mathbf{W}[\boldsymbol{v}]$ on the mean flow $u^{p}(V) \boldsymbol{e}_{p}$ yields

$$
\mathbf{W}\left[u^{p}(V) \boldsymbol{e}_{p}\right]=u^{p}(V) \mathbf{W}\left[\boldsymbol{e}_{p}\right]+\left(u^{p}(V)\right)^{\prime}\left(\nabla V: \boldsymbol{e}_{p}+\boldsymbol{e}_{p}: \nabla V\right)
$$

We define the matrix $\mathbf{W}^{\star}\left[\boldsymbol{e}_{p}\right]:=\nabla V: \boldsymbol{e}_{p}+\boldsymbol{e}_{p}: \nabla V$ and the tensor $\pi^{\star}\left[\boldsymbol{e}_{p}\right]=\pi\left[\mathbf{W} \longrightarrow \mathbf{W}^{\star}\left[\boldsymbol{e}_{p}\right]\right]$. $\pi^{\star}$ has the same structure as the viscous tensor $\pi$ except that the rate of strain tensor is replaced by $\mathbf{W}^{\star}$. This allows us to write the viscous tensor in the form

$$
\begin{aligned}
& \pi_{k}\left[u_{k}^{p}(V) \boldsymbol{e}_{p}\right]=u_{k}^{p}(V) \pi_{k}\left[\boldsymbol{e}_{p}\right]+\frac{u_{k}^{p}(V)}{d V} \pi_{k}^{\star}\left[\boldsymbol{e}_{p}\right] \\
& \pi_{k}\left[u_{k}^{t}(V) \boldsymbol{e}_{t}\right]=u_{k}^{t}(V) \pi_{k}\left[\boldsymbol{e}_{t}\right]+\frac{u_{k}^{t}(V)}{d V} \pi_{k}^{\star}\left[\boldsymbol{e}_{t}\right]
\end{aligned}
$$

$\pi_{k}\left[\boldsymbol{e}_{p}\right]$ and $\pi_{k}^{\star}\left[\boldsymbol{e}_{p}\right]$ only depend on the geometry of the configuration and the viscous coefficients. Inserting this decomposition into Eq. (54) makes the poloidal viscous force a second order differential operator on $u_{k}^{p}$ and $u_{k}^{t}$. The poloidal viscous force is

$$
\begin{aligned}
<\boldsymbol{e}_{p} \cdot \nabla \cdot \pi_{k}\left[u^{p}(V) \boldsymbol{e}_{p}\right]> & =\frac{d}{d V}\left(\frac{u_{k}^{p}(V)}{d V}<\boldsymbol{e}_{p} \cdot \pi_{k}^{\star}\left[\boldsymbol{e}_{p}\right] \cdot \nabla V>+u_{k}^{p}(V)<\boldsymbol{e}_{p} \cdot \pi_{k}\left[\boldsymbol{e}_{p}\right] \cdot \nabla V>\right) \\
& -\left(\frac{u_{k}^{p}(V)}{d V}<\pi_{k}^{\star}\left[\boldsymbol{e}_{p}\right] \cdot \nabla: \boldsymbol{e}_{p}>+u_{k}^{p}(V)<\pi_{k}\left[\boldsymbol{e}_{p}\right] \cdot \nabla: \boldsymbol{e}_{p}>\right)
\end{aligned}
$$

The right hand side defines an operator $L_{p p}$ by $<\boldsymbol{e}_{p} \cdot \nabla \cdot \pi_{k}\left[u^{p}(V) \boldsymbol{e}_{p}\right]>:=L_{p p} u_{k}^{p}$. In the same way we define $\left\langle\boldsymbol{e}_{p} \cdot \nabla \cdot \pi_{k}\left[u^{t}(V) \boldsymbol{e}_{t}\right]\right\rangle:=L_{p t} u_{k}^{p},\left\langle\boldsymbol{e}_{t} \cdot \nabla \cdot \pi_{k}\left[u^{p}(V) \boldsymbol{e}_{p}\right]\right\rangle:=L_{t p} u_{k}^{p}$ and $<\boldsymbol{e}_{t} \cdot \nabla \cdot \pi_{k}\left[u^{t}(V) \boldsymbol{e}_{t}\right]>:=L_{t t} u_{k}^{t}$, which allows us to write the viscous forces in the matrix form

$$
\left(\begin{array}{c}
<\boldsymbol{e}_{p} \cdot \nabla \cdot \pi_{k}> \\
<\boldsymbol{e}_{t} \cdot \nabla \cdot \pi_{k}>
\end{array}\right)=\left(\begin{array}{ll}
L_{k, p p} & L_{k, p t} \\
L_{k, t p} & L_{k, t t}
\end{array}\right)\left(\begin{array}{c}
u^{p}(V) \\
u^{t}(V)
\end{array}\right)
$$

In this form the viscous forces are valid in any cylindrical geometry or toroidal geometry. In cylinder geometry all magnetic pumping effects are zero and the tensors $\nabla: \boldsymbol{e}_{t}$ and $\pi_{k}\left[\boldsymbol{e}_{t}\right]$ are zero. This cancels several coefficients in $L_{p t}, L_{t p}, L_{t t}$. Often a slab geometry is used to study the evolution of drift waves and zonal flow; in this geometry all coordinates are Cartesian and the viscous operators are reduced to

$$
L_{i k}=\frac{d}{d z}\left(<\boldsymbol{e}_{i} \cdot \pi_{k}^{\star}\left[\boldsymbol{e}_{k}\right] \cdot \nabla z>\frac{d}{d z}\right) \quad ; \quad i, k=x, y
$$

$z$ is the direction of the plasma gradients.

The special properties of the base vectors in cylinder geometry lead to a significant reduction of the coeffient in the viscous operators. The axial base vector $\boldsymbol{e}_{t}$ is a constant vector and therefore $\pi_{k}\left[\boldsymbol{e}_{t}\right]=0$. The poloidal base vector $\boldsymbol{e}_{p} ;\left|\boldsymbol{e}_{p}\right|=r$ can be interpreted as a rigid rotation with zero rate of strain tensor and consequently we get $\pi_{k}\left[\boldsymbol{e}_{p}\right]=0$. This implies that the shear of the poloidal rotation is the derivative of $u_{k}^{p}(V)$; every particle species has its own velocity shear. This is the result of $\boldsymbol{e}_{p}=\{-y, x, 0\} \longrightarrow \nabla: \boldsymbol{e}_{p}+\left(\nabla: \boldsymbol{e}_{p}\right)^{\top}=0$. Since $\nabla: \boldsymbol{e}_{p}$ is an antisymmetric tensor and $\pi^{\star}$ is symmetric the term $\pi^{\star} \cdot \nabla: \boldsymbol{e}_{p}$ is zero, too. Finally, we can write the viscous operator in cylinder geometry

$$
L_{i k}=\frac{d}{d V}\left(<\boldsymbol{e}_{i} \cdot \pi_{k}^{\star}\left[\boldsymbol{e}_{k}\right] \cdot \nabla V>\frac{d}{d V}\right) \quad ; \quad i, k=p, t
$$


The coefficients of the diagonal operators $L_{p p}, L_{t t}$ depend only on the shear viscosity because of

$$
\boldsymbol{e}_{i} \cdot \pi_{k}^{\star}\left[\boldsymbol{e}_{i}\right] \cdot \nabla V=\boldsymbol{e}_{i}: \nabla V \cdot \pi_{k}^{\star}\left[\boldsymbol{e}_{i}\right]=-\sum_{l} \eta_{l} \mathbf{W}^{\star} \mathbf{A}_{l} \mathbf{W}^{\star}
$$

The surface-averaged shear viscosity grows strongly with the radius because of $\mathbf{W}^{\star} \mathbf{A}_{l} \mathbf{W}^{\star} \approx V^{2}$ we find

$$
<\boldsymbol{e}_{p} \cdot \pi_{k}^{\star}\left[\boldsymbol{e}_{p}\right] \cdot \nabla V>\approx \eta_{1, k} V^{2} \quad ; \quad<\boldsymbol{e}_{t} \cdot \pi_{k}^{\star}\left[\boldsymbol{e}_{t}\right] \cdot \nabla V>\approx \eta_{1, k} V
$$

$\eta_{1, k}$ is the coefficient of shear viscosity. As already mentioned previously, this sum has no contributions from the gyroviscosity. However, this is not true for the non-diagonal terms; these coupling terms are dominated by the gyro viscosity. The gyro viscosity does not contribute to the slowing down of the poloidal and axial flow, its main effect is to exchange momentum between poloidal and axial directions.

Surface-averaged inertial forces in general geometry have been investigated in [27]; in this paper also the correlation to the paper of Hassam and Drake [31] has been pointed out. This concerns mainly the normal and the anomalous Stringer spin-up. Cylindrical geometry alleviates the analysis owing to the absence of poloidal asymmetries and Pfirsch-Schlüter currents. Averaging the inertial force over the magnetic surface yields

$$
<\boldsymbol{e}_{p} \cdot \nabla \cdot n \boldsymbol{v}: \boldsymbol{v}>=\frac{d}{d V} \int \boldsymbol{e}_{p} \cdot \boldsymbol{v} n \boldsymbol{v} \cdot d \boldsymbol{f}-<n \boldsymbol{v}: \boldsymbol{v} \cdot \nabla: \boldsymbol{e}_{p}>
$$

Because of the antisymmetry of $\nabla: \boldsymbol{e}_{p}$ the last term is zero. Since the base vectors in cylinder geometry are mutually orthogonal we get $\boldsymbol{e}_{p} \cdot \boldsymbol{v}=u^{p} \boldsymbol{e}_{p} \cdot \boldsymbol{e}_{p}$. Note, that this distinguishes the cylinder from a torus where the scalar product $\boldsymbol{e}_{p} \cdot \boldsymbol{e}_{t} \neq 0$ is non-zero and the inertial forces provide a coupling between poloidal and toroidal mean flow [27]. In a next step we perform the averaging in time which yields

$$
\begin{aligned}
& <\boldsymbol{e}_{p} \cdot \nabla \cdot \overline{n \boldsymbol{v}: \boldsymbol{v}}>=\frac{d}{d V} \boldsymbol{e}_{p}^{2} u^{p}(V) \int \overline{n \boldsymbol{v}} \cdot d \boldsymbol{f}+\frac{d}{d V} \boldsymbol{e}_{p}^{2} \int \overline{\delta u^{p} \delta(n \boldsymbol{v})} \cdot d \boldsymbol{f} \\
& <\boldsymbol{e}_{t} \cdot \nabla \cdot \overline{n \boldsymbol{v}: \boldsymbol{v}}>=\frac{d}{d V} \boldsymbol{e}_{t}^{2} u^{t}(V) \int \overline{n \boldsymbol{v}} \cdot d \boldsymbol{f}+\frac{d}{d V} \boldsymbol{e}_{p}^{2} \int \overline{\delta u^{p} \delta(n \boldsymbol{v})} \cdot d \boldsymbol{f}
\end{aligned}
$$

The relation between contravariant and covariant components of the velocity is $\boldsymbol{e}_{p}^{2} u^{p}=u_{p}, \boldsymbol{e}_{t}^{2} u^{p}=$ $u_{t}$. In order to shorten the notation we introduce several abbreviations: $\left.\eta_{i k}:=<\boldsymbol{e}_{i} \cdot \pi_{k}^{\star}\left[\boldsymbol{e}_{k}\right] \cdot \nabla V\right\rangle$ and

$$
R^{p}=\boldsymbol{e}_{p}^{2} \int \overline{\delta u^{p} \delta(n \boldsymbol{v})} \cdot d \boldsymbol{f} \quad ; \quad R^{t}=\boldsymbol{e}_{t}^{2} \int \overline{\delta u^{t} \delta(n \boldsymbol{v})} \cdot d \boldsymbol{f}
$$

The viscous operator is in matrix form

$$
L_{k}=-\frac{d}{d V}\left(\begin{array}{cc}
\eta_{k, p p} & \eta_{k, p t} \\
\eta_{k, t p} & \eta_{k, t t}
\end{array}\right) \frac{d}{d V}
$$

and the Reynolds averaged equations in matrix form are

$$
\begin{aligned}
L_{k}\left(\begin{array}{c}
u_{k}^{p} \\
u_{k}^{t}
\end{array}\right) & +\frac{m_{k}}{m_{e}} \frac{\delta_{e}^{2}}{a^{2}} \frac{d}{d V}\left(\begin{array}{c}
F_{k} \boldsymbol{e}_{p}^{2} u_{k}^{p}+\boldsymbol{e}_{p}^{2} R_{k}^{p} \\
F_{k} \boldsymbol{e}_{t}^{2} u_{k}^{t}+\boldsymbol{e}_{t}^{2} R_{k}^{t}
\end{array}\right) \\
& =\sigma_{k} F_{k}\left(\begin{array}{c}
b^{t}(V) \\
b^{p}(V)
\end{array}\right)+\frac{\nu_{e}}{\Omega_{e}} \sum_{i} f_{i k}\left(\begin{array}{c}
\boldsymbol{e}_{p}^{2} u_{k}^{p} \\
\boldsymbol{e}_{t}^{2} u_{k}^{t}
\end{array}\right) \\
& +\sigma_{k}\left(\begin{array}{c}
<\overline{\delta n_{k} \boldsymbol{e}_{p} \cdot \delta \boldsymbol{E}}> \\
<\overline{\delta n_{k} \boldsymbol{e}_{t} \cdot \delta \boldsymbol{E}}>
\end{array}\right)+\sigma_{k}\left(\begin{array}{c}
0 \\
<\bar{n}_{k} \boldsymbol{e}_{t} \cdot \overline{\boldsymbol{E}}>
\end{array}\right)
\end{aligned}
$$


The overbar denotes an average over the longest time scale in the turbulent plasma; this annihilates any time derivative in the equations. If one restricts the averaging to the fast time scale of drift waves the equations describe the evolution of zonal flow. In the literature the zonal flow is defined as the $m=0, n=0$ component of the $\boldsymbol{E} \times \boldsymbol{B}$-velocity; the electric potential depends only on the surface label and the time. This velocity is common for all particle species and does not describe the full velocity of each particle species. The zonal flow defined this way does not contribute to the diamagnetic current. A natural generalisation of the concept of zonal flow in a multi-species plasma is to define zonal flow for every particle species separately and to understand zonal flow as the surface-averaged tangential velocity. However, regardless of what is called zonal flow the spin-up equations of the tangential particle fluxes are

$$
\begin{aligned}
& \frac{m_{k}}{m_{e}} \frac{\partial}{\partial \tau}\left(\begin{array}{c}
<n_{k} \boldsymbol{e}_{p} \cdot \boldsymbol{u}_{k}> \\
<n_{k} \boldsymbol{e}_{t} \cdot \boldsymbol{u}_{k}>
\end{array}\right)+L_{k}\left(\begin{array}{c}
u_{k}^{p} \\
u_{k}^{t}
\end{array}\right)+\frac{m_{k}}{m_{e}} \frac{\delta_{e}^{2}}{a^{2}} \frac{d}{d V}\left(\begin{array}{c}
F_{k} \boldsymbol{e}_{p}^{2} u_{k}^{p}+\boldsymbol{e}_{p}^{2} R_{k}^{p} \\
F_{k} \boldsymbol{e}_{t}^{2} u_{k}^{t}+\boldsymbol{e}_{t}^{2} R_{k}^{t}
\end{array}\right) \\
= & \sigma_{k} F_{k}\left(\begin{array}{c}
b^{t}(V) \\
b^{p}(V)
\end{array}\right)+\frac{\nu_{e}}{\Omega_{e}} \sum_{i} f_{i k}\left(\begin{array}{c}
\boldsymbol{e}_{p}^{2} u_{k}^{p} \\
\boldsymbol{e}_{t}^{2} u_{k}^{t}
\end{array}\right)+\sigma_{k}\left(\begin{array}{c}
<\overline{\delta n_{k} \boldsymbol{e}_{p} \cdot \delta \boldsymbol{E}}> \\
<\overline{\delta n_{k} \boldsymbol{e}_{t} \cdot \delta \boldsymbol{E}>}
\end{array}\right) \\
+ & \sigma_{k}\left(\begin{array}{c}
0 \\
<\bar{n}_{k} \boldsymbol{e}_{t} \cdot \overline{\boldsymbol{E}}>
\end{array}\right)
\end{aligned}
$$

The components of the tangential flow are time-dependent $u_{k}^{p}(V, \tau), u_{k}^{t}(V, \tau)$ which we can write as $u_{k}^{p}(V, \tau)=U_{k}^{p}(V)+\delta u_{k}^{p}(V, \tau)$ and the same decomposition for $u_{k}^{t}(V, \tau)$. $U_{k}^{p}(V)$ is the mean flow, averaged over the long time scale. In order to proceed, we invoke the so-called Boussinesq approximations which replaces the density $n_{k}$ by the surface averaged density $N_{k}(V, \tau)$ which implies $<n_{k} \boldsymbol{e}_{p} \cdot \boldsymbol{u}_{k}>=\boldsymbol{e}_{p}^{2} N_{k} u_{k}^{p}(V, \tau)$. The surface-averaged particle flux is the product of the surface-averaged density and the surface-averaged velocity. The surface-averaged equation of continuity is

$$
\frac{\partial N_{k}}{d \tau}+\frac{\delta_{e}^{2}}{a^{2}} \frac{d}{d V} \int \bar{n}_{k} \boldsymbol{v}_{k} \cdot d \boldsymbol{f}=<s_{k}>
$$

which leads to

$$
\begin{aligned}
& \frac{\partial N_{k} u_{k}^{p}}{\partial \tau}=N_{k} \frac{\partial u_{k}^{p}}{d \tau}+<s_{k}>u_{k}^{p}-\frac{\delta_{e}^{2}}{a^{2}} u_{k}^{p} \frac{d F_{k}}{d V} \\
& \frac{\partial N_{k} u_{k}^{t}}{\partial \tau}=N_{k} \frac{\partial u_{k}^{t}}{d \tau}+<s_{k}>u_{k}^{t}-\frac{\delta_{e}^{2}}{a^{2}} u_{k}^{t} \frac{d F_{k}}{d V}
\end{aligned}
$$

Inserting these terms into the Eqs. (70) yields

$$
\begin{aligned}
& \frac{m_{k}}{m_{e}} N_{k} \frac{\partial}{\partial \tau}\left(\begin{array}{c}
\boldsymbol{e}_{p}^{2} u_{k}^{p} \\
\boldsymbol{e}_{t}^{2} u_{k}^{t}
\end{array}\right)+\frac{m_{k}}{m_{e}}<s_{k}>\left(\begin{array}{c}
\boldsymbol{e}_{p}^{2} u_{k}^{p} \\
\boldsymbol{e}_{t}^{2} u_{k}^{t}
\end{array}\right)+\frac{m_{k}}{m_{e}} \frac{\delta_{e}^{2}}{a^{2}} F_{k} \frac{d}{d V}\left(\begin{array}{c}
\boldsymbol{e}_{p}^{2} u_{k}^{p} \\
\boldsymbol{e}_{t}^{2} u_{k}^{t}
\end{array}\right) \\
+ & L_{k}\left(\begin{array}{c}
u_{k}^{p} \\
u_{k}^{t}
\end{array}\right)+\frac{m_{k}}{m_{e}} \frac{\delta_{e}^{2}}{a^{2}} \frac{d}{d V}\left(\begin{array}{c}
\boldsymbol{e}_{p}^{2} R_{k}^{p} \\
\boldsymbol{e}_{t}^{2} R_{k}^{t}
\end{array}\right)=\sigma_{k} F_{k}\left(\begin{array}{c}
b^{t}(V) \\
b^{p}(V)
\end{array}\right)+\frac{\nu_{e}}{\Omega_{e}} \sum_{i} f_{i k}\left(\begin{array}{c}
\boldsymbol{e}_{p}^{2} u_{k}^{p} \\
\boldsymbol{e}_{t}^{2} u_{k}^{t}
\end{array}\right) \\
+ & \sigma_{k}\left(\begin{array}{c}
<\overline{\delta n_{k} \boldsymbol{e}_{p} \cdot \delta \boldsymbol{E}}> \\
<\overline{\delta n_{k} \boldsymbol{e}_{t} \cdot \delta \boldsymbol{E}}>
\end{array}\right)+\sigma_{k}\left(\begin{array}{c}
<\bar{n}_{k} \boldsymbol{e}_{t} \cdot \overline{\boldsymbol{E}}>
\end{array}\right)
\end{aligned}
$$

As already mentioned, in a hydrogen plasma with cold gas refueling the source terms $s_{k}$ and the net flux $F_{k}$ are limited to the boundary region. Further inside, these terms are zero and besides the friction and viscous forces only the turbulent terms are left. Viscosity and friction slow down plasma motion, while the turbulent terms may lead to spin-up. The poloidal forces $\sigma_{k}<\overline{\delta n_{k} \boldsymbol{e}_{p} \cdot \delta \boldsymbol{E}}>$ drive electrons and ions in opposite direction and due to the quasi-neutrality 
of drift waves the sum is zero. The same result holds for the axial electric force $\sigma_{k}<\bar{n}_{k} \boldsymbol{e}_{t} \cdot \overline{\boldsymbol{E}}>$. In order to make the system of equations complete we average the radial force balance (48) over the magnetic surface

$$
\frac{d}{d V} \beta_{k}(V, \tau)=\sigma_{k}<n_{k} \boldsymbol{e}_{V} n_{k} \boldsymbol{E}>+\sigma_{k}\left(b^{t}(V)<n_{k} u_{p, k}>-b^{p}(V)<n_{k} u_{t, k}>\right.
$$

Invoking the Boussinesq approximation again $n_{k} \longrightarrow N_{k}(V, \tau)$ this is

$$
\frac{d}{d V} \beta_{k}(V, \tau)=-\sigma_{k} N_{k} \frac{d \Phi}{d V}+\sigma_{k} N_{k}\left(-b^{t}(V) u_{k}^{p}+b^{p}(V) u_{k}^{t}\right)
$$

$\Phi(V, \tau)$ is the surface-averaged electric potential which in the literature is considered as the key element of zonal flow. In the sum over all particle species the electric field drops out and the pressure gradient is balanced by the poloidal and toroidal (axial) diamagnetic currents.

In this approximation, the radial force balance is valid also in any toroidal system. This radial force can be considered as the instantaneous radial balance between pressure gradient, Lorentz force and radial electric field. How to treat this equation? Often this equation is used to write the poloidal velocity in terms of the diamagnetic drift and the electric drift, which requires another equation to compute the parallel velocity or $u_{t, k}$. Here the standpoint is to compute the density profile and the radial electric field, if the temperature and the two velocity components are given. The computation of the temperature profile needs consideration of the energy balance. However, since the numerical application is focussed on the transition to the $\mathrm{HDH}$-mode in Wendelstein 7AS, where the temperature profiles stay nearly constant during the transition, the energy balance can be postponed.

\section{The problem of closure}

Let us return to the Reynolds-averaged equations for the mean velocities (68). The turbulent shear stress $R_{k}^{p}, R_{k}^{t}$ originates from the non-diagonal terms of the Reynolds stress tensor which describes the radial momentum transfer by turbulent eddies. These shear stresses must be either computed separately by solving the time-dependent equations or by an empirical ansatz which summarises the effect of turbulence on the mean flow. In fluid dynamics this has led to the concept of eddy viscosity. In atmospheric physics this turbulent viscosity or eddy diffusion coefficient plays the dominant role in forming the profile of the velocity shear. Such an eddy diffusion coefficient has also been introduced in the theory of the super-rotation on Venus [32]. In fluid theory the general ansatz is to assume a linear relation between shear stress and the rate of strain tensor [23], [33]. In a highly anisotropic plasma this certainly would lead to a complicated tensorial ansatz as the example of the Braginskii viscosity shows. In the cylindrical geometry the ansatz is simpler since only two orthogonal directions need to be considered. The inertial terms $R_{k}^{p}$ can be interpreted as the turbulent transport of poloidal momentum by radial fluctuation, the turbulent transport of a passive scalar. The result of turbulent momentum exchange is a reduction of the velocity shear until the flow is a rigid rotation. As in hydrodynamics [23] this turbulent momentum flux is set proportional to the shear of the mean poloidal and axial velocity. Likewise, the ansatz will be made for the radial transport of axial momentum

$$
R^{p}=\boldsymbol{e}_{p}^{2} \int \overline{\delta u^{p} \delta(n \boldsymbol{v})} \cdot d \boldsymbol{f}=-\boldsymbol{e}_{p}^{2} \eta^{p} \frac{d u^{p}}{d V} \quad ; \quad R^{t}=\boldsymbol{e}_{t}^{2} \int \overline{\delta u^{t} \delta(n \boldsymbol{v})} \cdot d \boldsymbol{f}=-\boldsymbol{e}_{t}^{2} \eta^{t} \frac{d u^{t}}{d V}
$$

$\eta^{p}, \eta^{t}$ are the eddy viscosity coefficients of turbulent momentum transport in dimensionless form. All details of turbulence are summarized in these coeffcients and with a growing level of turbulence these coefficients will grow, too. As seen from the definition of the turbulent Reynolds 
stress, it is the radial component of the drift waves which leads to anomalous momentum transport together with the poloidal fluctuations $\delta u^{p}$. This component consists of the zonal flow (the surface averaged part of $\delta u^{p}$, slow time scale) and the $\delta u^{p}$ of the drift waves (fast time scale). Since $\overline{\delta(n \boldsymbol{u})} \cdot d \boldsymbol{f}=0$ on the fast time scale, the zonal flow does not contribute to the momentum transport. There is no immediate effect of the zonal flow on the Reynolds stress. If however, the shear of zonal flow or mean flow leads to a decorrelation of the poloidal and radial drift wave fluctuations, the Reynolds stresses will be diminuished which implies a reduction of the eddy viscosity $\eta^{p}$. In a similar way any decorrelation in $\overline{\delta u_{t} \delta(n \boldsymbol{u})} \cdot d \boldsymbol{f}$ leads to a reduction of $\eta^{t}$. This effect has been used to model anomalous transport coefficients as a decreasing function of velocity shear, where velocity shear is meant as the shear of the electric drift [1]. In contrast to collisional viscosity, the sign of the eddy viscosity coefficients are not fixed; depending on the correlation of drift wave eddies the sign can be either positive or negative.

Eddy viscosity in MHD turbulence has been computed numerically by Kim et al. [34]. Sugama and Horton [35] used quasi-linear theory to compute anomalous momentum transport due to electrostatic turbulence. The anomalous momentum flux is proportional to the shear of the toroidal velocity: they called the coefficient anomalous viscosity. Shaing et al.[36] [37] have computed an anomalous toroidal ion viscosity which depends on the power spectrum of the electric field fluctuations. Staebler and Dominguez [38] started from slab geometry and derived an anomalous viscosity as a response to electrostatic fluctuations. The dimension of the eddy viscosity is $\eta^{p}=\lambda^{2} \nu_{t}$ where $\lambda$ is a length scale of the turbulence and $\nu_{t}$ a characteristic frequency.

The coupling between density fluctuations and electric field fluctuations gives rise to a poloidal force $\left\langle\overline{\delta n_{k} \boldsymbol{e}_{p} \cdot \delta \boldsymbol{E}}>\right.$ which is equivalent to a radial anomalous particle flux. If we consider this term and its axial partner as given, the system (68) can be solved as in the case of a quiescent plasma. A more satisfying procedure would be to model these forces as has been done with the viscous forces. Because of the quasi-neutrality, the sum of these anomalous poloidal and axial $\sigma_{k} \delta n_{k} \delta \boldsymbol{E}$-forces is zero which means that the sum has no effect on the total momentum of the poloidal or axial flow. The fluctuations, however, lead to momentum exchange among the particle species. Thus, these fluctuations play the same role as the Coulomb collisions. Staebler [39] has studied the effect of drift wave turbulence starting from the Fokker-Planck equation; there it has been shown that the turbulent forces conserve momentum. Often the ansatz is made that the anomalous fluxes are proportional to the gradients which drive the instability defining this way an anomalous transport coefficient. Quasi-linear theory [40][35] has verified such linear relations between gradients and anomalous fluxes where the coefficients depend on the square of the electric field fluctuations. Since via the radial force balance the gradients and the com-

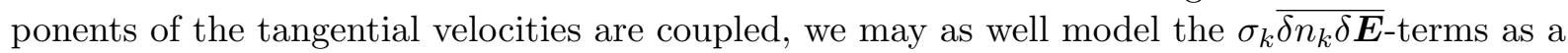
linear combination of all tangential velocity components.

$$
<\boldsymbol{t} \cdot \sigma_{k} \overline{\delta n_{k} \delta \boldsymbol{E}}>=\frac{\nu_{e}}{\Omega_{e}} \sum_{i}<\boldsymbol{t} \cdot \gamma_{k i} \boldsymbol{u}_{i}>\quad ; \quad \boldsymbol{t}=\boldsymbol{e}_{p}, \boldsymbol{e}_{t}
$$

Such a form of the anomalous particle fluxes has been found by Shaing [41] for electrostatic turbulence. This paper also applies to stellarators and, as in the case of neoclassical plateau diffusion, strong resonance effects at rational values of the rotational transform were found. Quasi-linear theory computes the response of the plasma on electrostatic fluctuations starting from a linearized kinetic equation. The lowest order distribution function is expanded in terms of the vectorial moments which are the mean velocity and the mean heat flux. Hence the natural result of quasi-linear theory is a linear relation between anomalous fluxes and the components of mean flow velocity [41]. After defining thermodynamic forces, these relations can be used to define anomalous transport coefficients.

Instead of anomalous transport coefficients, we have defined anomalous friction coefficients; 
the factor $\nu_{e} / \Omega_{e}$ has been introduced in order to make $\gamma_{k i}$ comparable to $f_{i k}$. These coefficients $\gamma_{k i}$ must satisfy the condition $\sum_{k} \gamma_{k i}=0 \forall i$. The enhanced momentum exchange between the plasma components can be described by an enhanced collision frequency. However, this simple ansatz does not account for the asymmetry of drift wave turbulence with a small wave vector parallel to the magnetic field and a large wave vector in perpendicular direction which implies that the toroidal $\delta \boldsymbol{E}$-force is smaller than the poloidal force. Therefore we consider $\gamma_{k i}^{l m}, l, m=p, t$ as a $2 \times 2$ matrix $\gamma^{l m}$ which describes this asymmetry.

$$
\begin{aligned}
& <\boldsymbol{e}_{p} \cdot \sigma_{k} \overline{\delta n_{k} \delta \boldsymbol{E}}>=\frac{\nu_{e}}{\Omega_{e}} \sum_{i}\left(\gamma_{i k}^{p p} u_{p, i}+\gamma_{i k}^{p t} u_{t, i}\right) \\
& <\boldsymbol{e}_{t} \cdot \sigma_{k} \overline{\delta n_{k} \delta \boldsymbol{E}}>=\frac{\nu_{e}}{\Omega_{e}} \sum_{i}\left(\gamma_{i k}^{t p} u_{p, i}+\gamma_{i k}^{t t} u_{t, i}\right)
\end{aligned}
$$

In general, the matrices $\gamma$ are not symmetric, in contrast to the matrix of Coulomb friction $f_{i k}$. Any decorrelation of drift waves eddies by shear flow [30] would reduce these anomalous friction coefficients. However, it should be noted that this is not the most general ansatz since temperature gradients and the associated tangential heat fluxes are also driving instabilities (ITG-modes etc). As in the case of Coulomb friction forces described above, the most general ansatz has linear terms in the heat fluxes, too. Such linear dependence of the anomalous particle fluxes on the poloidal and toroidal heat fluxes has been found by Shaing [42].

The Coulomb friction forces are symmetric and lead to a positive entropy production rate. Due to the symmetry, the friction forces are proportional to the difference of the particle velocities $\boldsymbol{u}_{i}-\boldsymbol{u}_{k}$, and, taking into account the radial force balance, proportional to the difference of radial gradients. This is the key to the accumulation of impurities which are diffusing into the plasma by interaction with the hydrogen background. In a torus, the Pfirsch-Schlüter factor enhances the effect.

Collecting all models and assumptions, the Reynolds averaged equations are

$$
\begin{aligned}
L_{k}\left(\begin{array}{c}
u_{k}^{p} \\
u_{k}^{t}
\end{array}\right) & -\frac{m_{k}}{m_{e}} \frac{\delta_{e}^{2}}{a^{2}} \frac{d}{d V}\left(\begin{array}{cc}
\boldsymbol{e}_{p}^{2} \eta_{k}^{p} & 0 \\
0 & \boldsymbol{e}_{t}^{2} \eta_{k}^{t}
\end{array}\right) \frac{d}{d V}\left(\begin{array}{c}
u_{k}^{p} \\
u_{k}^{t}
\end{array}\right)+\frac{m_{k}}{m_{e}} \frac{\delta_{e}^{2}}{a^{2}} \frac{d}{d V}\left(\begin{array}{c}
F_{k} \boldsymbol{e}_{p}^{2} u_{k}^{p} \\
F_{k} \boldsymbol{e}_{t}^{2} u_{k}^{t}
\end{array}\right) \\
& =\sigma_{k} F_{k}\left(\begin{array}{c}
b^{t}(V) \\
b^{p}(V)
\end{array}\right)+\frac{\nu_{e}}{\Omega_{e}} \sum_{i} f_{i k}\left(\begin{array}{c}
\boldsymbol{e}_{p}^{2} u_{i}^{p} \\
\boldsymbol{e}_{t}^{2} u_{i}^{t}
\end{array}\right) \\
& +\frac{\nu_{e}}{\Omega_{e}} \sum_{i}\left(\gamma_{i k}\right)\left(\begin{array}{c}
\boldsymbol{e}_{p}^{2} u_{i}^{p} \\
\boldsymbol{e}_{t}^{2} u_{i}^{t}
\end{array}\right)+\sigma_{k}\left(\begin{array}{c}
0 \\
<\bar{n}_{k} \boldsymbol{e}_{t} \cdot \overline{\boldsymbol{E}}>
\end{array}\right)
\end{aligned}
$$

Since $\boldsymbol{e}_{p}^{2} u_{k}^{p}=u_{k, p}$ are the covariant components, we can also formulate the system in terms of these covariant components.

In summary, we have modeled the fluctuating $\delta \boldsymbol{E}$-forces with the help of anomalous friction coefficients and the Reynolds stresses in terms of the eddy viscosity. The matrix of eddy viscosities has only diagonal terms; the eddy viscosity competes with the Braginskii shear viscosity and if the radial mixing length of turbulence is larger than the gyro radius and the characteristic frequencies larger than the collision frequencies the eddy viscosity is the dominating term.

Before going into some details of the anomalous coefficients, we will have a closer look at the mathematical structure and the boundary conditions. The system is a linear second order and inhomogeneous system. The inhomogeneous terms are the fluxes $\mathrm{F}_{k}$ and the toroidal loop voltage. It was argued that in the friction forces, in the viscous force and in the anomalous terms, the contributions by the heat flux will be ignored. These would add just another inhomogeneous term and the solution with respect to the velocities would follow the same procedure as without these terms. In the plasma center $V=0$ the poloidal velocity $u_{p}$ should be zero since otherwise 
the angular velocity would go to infinity. The angular velocity $u^{p}$ can be finite in the center; here the natural boundary condition is $d u^{p} / d V=0$ at $V=0$. The axial velocity has an extremum in the center, the derivative is zero $d u^{t} / d V=0$. On the plasma boundary $V=V_{a}$ we impose either Dirichlet conditions $u^{p}=u^{t}=0$ (sticking conditions) or zero derivatives. This last condition fits to free flow on a last magnetic surface without contact to material walls (Neumann conditions). It should be pointed out that including the viscous terms allows one to satisfy boundary condition on the tangential velocities. In reality, the boundary conditions may not be as simple as the Dirichlet or Neumann conditions; it requires a careful analysis of the physics outside the last closed flux surface in order to specify the boundary conditions. Let us assume that all coefficients are known functions of the radial coordinate $V$. The linear inhomogeneous system has a unique solution if the homogeneous has no sulution but $\boldsymbol{u}=0$. Given the fluxes $F_{k}$ as integral over the sources and the loop voltage, the solutions $u_{k}^{p}$, $u_{k}^{t}$ will be inserted into the radial force balance, which computes the density profiles and the radial electric field. The new density profile leads to new source terms and new fluxes. The procedure will be repeated until the iteration converges. A numerical example will be presented at the end of the paper.

\subsection{Bifurcation}

The system contains two possible places of non-linearity; one is the anomalous friction represented by the matrix $\gamma$ and the second one is the eddy (or anomalous) viscosity. These nonlinearities can be the origin of bifurcation. Following the generally accepted idea that velocity shear quenches turbulence, these coefficients can be modeled as a decreasing function of velocity shear. Velocity shear is represented by the derivatives of $u_{k}^{p}(V)$ and $u_{k}^{t}(V)$ and the most general ansatz would be to make the anomalous coefficients a decreasing function of all these components. In a hydrogen plasma with a small amount of impurities the turbulence is governed by the main constituents and the anomalous coefficients only depend on the velocity shear of the hydrogen ions and electrons. If we approximate the velocity by the electric drift, we arrive at the concept widely used in the literature. In a fusion plasma there are at least three ion species with different masses and fractions. Does the suppression of electrostatic turbulence depend on the velocity shear of all ion species or only on the shear of the $\boldsymbol{E} \times \boldsymbol{B}$-drift common to all ? As discussed by Terry in his review article on turbulence suppression by flow shear [30], the loss of correlation arises from the turbulent advection by the $(\boldsymbol{v} \nabla) \boldsymbol{v}$-term. It is the shear of the mean velocity which tilts and quenches the turbulent eddies and thus reduces turbulent transport. A possible ansatz for the anomalous viscosity coefficients is

$$
\eta^{p}=\eta^{p}\left(V, g_{k}^{p}, g_{k}^{t}\right), \quad \eta^{t}=\eta^{t}\left(V, g_{k}^{p}, g_{k}^{t}\right), \gamma=\gamma\left(V, g_{i}^{p}, g_{i}^{t}\right), \quad(i=1, \ldots, K)
$$

where we introduced the notation $g_{k}^{p}, g_{k}^{t}$ for the radial derivatives of the velocities $u_{k}^{p}, u_{k}^{t}$. In this general ansatz the turbulent viscosity of particle species $k$ is a function of the velocity shear $g_{k}$ and the special case of $\boldsymbol{u}_{k} \longrightarrow \boldsymbol{E} \times \boldsymbol{B}$ is included. In view of the following argument, it is unimportant whether the anomalous friction coefficients depend on all derivatives of $u_{i}^{p}, u_{i}^{t}$ or not. The only restriction is that the anomalous coefficients are independent of the sign of the derivatives and monotonously decreasing. This decrease may even exhibit a step-like character. Adopting these kind of specifications, the system (81) is non-linear and second order. In order to avoid the inconveniences with the indices, we summarise the poloidal and axial velocities in a vector $\boldsymbol{f}_{k}=\left\{u_{k}^{p}, u_{k}^{t}\right\}$ and the derivatives in $\boldsymbol{g}_{k}=\left\{g_{k}^{p}, g_{k}^{t}\right\}$. Next, these 2-vectors are summarised in $\boldsymbol{f}=\left\{\boldsymbol{f}_{k}\right\}$ and $\boldsymbol{g}=\left\{\boldsymbol{g}_{k}\right\} . \boldsymbol{f}$ is a vector in a $K$-dimensional vector space $(K$-space) and its components are 2-dimensional vectors. $\boldsymbol{g}$ is the vector of velocity shear. The Braginskii viscosity is a $2 \times 2$-matrix

$$
\eta_{k}=\left(\begin{array}{cc}
\eta_{k, p p} & \eta_{k, p t} \\
\eta_{k, t p} & \eta_{k, t t}
\end{array}\right)
$$


which will be summarised in the diagonal matrix $\eta_{b}=\left\{\eta_{k} \delta_{i k}\right\}$. In the same way we define the diagonal matrix of anomalous viscosities

$$
E=\left(\begin{array}{cc}
\boldsymbol{e}_{p}^{2} & 0 \\
0 & \boldsymbol{e}_{t}^{2}
\end{array}\right) \quad ; \quad \eta_{a n, k}=\frac{m_{k}}{m_{e}} E\left(\begin{array}{cc}
\eta_{k}^{p} & 0 \\
0 & \eta_{k}^{t}
\end{array}\right) \longrightarrow \eta_{a n}=\left\{\eta_{a n, k} \delta_{i k}\right\}
$$

Furthermore, we define

$$
C=\left\{f_{i k} E\right\} \quad ; \quad C_{a n}=\left\{\gamma_{i k} E\right\} \quad ; \quad F=\left\{\sigma_{k} F_{k}\left(\begin{array}{c}
b^{t}(V) \\
b^{p}(V)
\end{array}\right)\right\}
$$

and

$$
G=\left\{\frac{m_{k}}{m_{e}} F_{k} E \delta_{i k}\right\} \quad ; \quad U_{\text {loop }}=\left\{\sigma_{k}\left(\begin{array}{c}
0 \\
<\bar{n}_{k} \boldsymbol{e}_{t} \cdot \bar{E}>
\end{array}\right)\right\}
$$

These abbreviations transfer the system (81) of $2 K$ equations into the form

$$
-\frac{d}{d V}\left(\eta_{b}+\frac{\delta_{e}^{2}}{a^{2}} \eta_{a n}\right) \frac{d \boldsymbol{f}}{d V}+\frac{\delta_{e}^{2}}{a^{2}} \frac{d}{d V} G \boldsymbol{f}=F+\frac{\nu_{e}}{\Omega_{e}}\left(C+C_{a n}\right) \boldsymbol{f}+U_{\text {loop }}
$$

The scalar product in $K$-space is $\boldsymbol{a} \cdot \boldsymbol{b}=\sum_{k} a_{k} b_{k}$, where $a_{k} b_{k}$ are the scalar products in a 2 -dimensional vector space. Without the anomalous terms the system is linear and presents no reason for bifurcations.

In order to demonstrate how the anomalous transport coefficient can give rise to bifurcation give a simple example which has the same structure as the system above. Let $[0 \leq x \leq 1]$ be a domain in $x$ and

$$
-\frac{d}{d x} \eta\left(u_{x}\right) \frac{d u}{d x}+D\left(u_{x}\right) u=\lambda h(x) \quad ; \quad u_{x}=\frac{d u}{d x} \quad ; \quad u(0)=u(1)=0 \quad ; \quad \lambda>0
$$

$\eta$ and $D$ are positive and continuous functions of $u_{x} . h(x)$ is a given continuous source function and $\lambda$ a control parameter characterising the level of the source term. It can easily be shown that any solution is bounded and has bounded derivatives. The upper bounds are proportional to $\lambda / m$, where $m$ is the minimum of $\eta_{0}, D_{0}$. With $\lambda \longrightarrow 0$ the equation is homogeneous and has no finite solution. The standard procedure of testing for bifurcation is linearisation around a solution $U(x, \lambda)$ and searching for solutions $\delta u$ of the linearized problem [43].

$$
-\frac{d}{d x}\left(\eta+\eta^{\prime} U_{x}\right) \frac{d \delta u}{d x}+D\left(U_{x}\right) \delta u+D^{\prime} U \frac{d \delta u}{d x}=0 \quad ; \quad \delta u(0)=\delta u(1)=0
$$

The integral relation for $\delta u$ is

$$
\int_{0}^{1}\left\{\left[\eta+\eta^{\prime} u_{x}\right]\left(\frac{d \delta u}{d x}\right)^{2}+\left[D-\frac{1}{2} \frac{d}{d x}\left(u D^{\prime}\right)\right](\delta u)^{2}\right\} d x=0
$$

The prime denotes the derivation with respect to $u_{x}$. A sufficient condition for the absence of bifurcation is

$$
\eta+\eta^{\prime} u_{x}>0 \text { and } D-\frac{1}{2} \frac{d}{d x}\left(u D^{\prime}\right)>0
$$

The violation of one of these conditions is a necessary condition for the existence of bifurcation. As an example for $\eta$ and $D$ we propose

$$
\eta=\eta_{0}+\frac{\eta_{1}}{1+\alpha_{1} u_{x}^{2}} \quad ; \quad D=D_{0}+\frac{D_{1}}{1+\alpha_{2} u_{x}^{2}}
$$


$\eta_{0}, \eta_{1}, D_{0}, D_{1}, \alpha_{1}, \alpha_{2}$ are positive constants. The conditions for absence of bifurcations are

$$
\left.\eta_{0}+\frac{\eta_{1}}{1+\alpha_{1} u_{x}^{2}}-\frac{2 \eta_{1} \alpha_{1} u_{x}^{2}}{\left(1+\alpha_{1} u_{x}^{2}\right.}\right)^{2}>0 \quad ; \quad D_{0}+\frac{D_{1}}{\left(1+\alpha_{2} u_{x}^{2}\right)}+\frac{d}{d x} \frac{D_{1} u u_{x} \alpha_{2}}{\left(1+\alpha_{2} u_{x}^{2}\right)^{2}}>0
$$

Setting these terms equal to zero defines a surface in the space of the control parameters $\lambda, \alpha_{1}, \alpha_{2}$; a surface on which multiple solutions bifurcate. Some general conclusions can be made

- Both non-linear viscosity and non-linear diffusion coefficients can lead to bifurcation

- The point of bifurcation is the point where the linearised problem has an eigenvalue equal to zero; the inequalities given in (91) are sufficient conditions for the absence of bifurcation.

- If $\alpha_{1}$ and $\alpha_{2}$ are small enough there is no bifurcation

- There is a threshold in $\lambda$. Below this threshold bifurcation does not occur

Since the source is the particle flux - or integrated particle source - this would restrict the phenomenon of bifurcation to the boundary layer determined by the penetration length of neutrals. However, here one should keep in mind that all effects related to the heat flux have been ignored in the momentum balance. Retaining this terms would add extra source terms and couple the momentum balance to the energy balance.

The conditions derived above can be extended to the system (87) of coupled equations. We consider the hydrogen refueling rate as control parameter and write the particle flux term as $\lambda F$ where $\lambda$ is a diagonal matrix $\lambda_{k} \delta_{i k}$. We assume that a solution $\boldsymbol{f}_{0}\left(V, \lambda_{H}\right)$ exists, which satisfies the boundary conditions. $\lambda_{H}$ is proportional to the hydrogen flux and the solution exists in a domain $\lambda_{0}<\lambda_{H}<\lambda_{1}$. The variational equation [43] is

$$
-\frac{\delta_{e}^{2}}{a^{2}} \frac{d}{d V}\left(\eta_{b}+\eta_{a n}\left(\boldsymbol{g}_{0}\right)+\boldsymbol{g}_{0} \eta_{a n}^{\prime}\right) \frac{d \delta \boldsymbol{f}}{d V}+\frac{\delta_{e}^{2}}{a^{2}} \frac{d}{d V} G \delta \boldsymbol{f}=\frac{\nu_{e}}{\Omega_{e}}\left(C+C_{a n}\left(\boldsymbol{g}_{0}\right) \delta \boldsymbol{f}+\frac{\nu_{e}}{\Omega_{e}} C^{\prime}\left(\boldsymbol{g}_{0}\right) \boldsymbol{f}_{0} \frac{d \delta \boldsymbol{f}}{d V}\right.
$$

Here, $\boldsymbol{g}_{0} \eta_{a n}^{\prime}$ and $C^{\prime}\left(\boldsymbol{g}_{0}\right) \boldsymbol{f}_{0}$ are matrices which occur when the system is linearised: $\eta_{a n}\left(\boldsymbol{g}_{0}+\delta \boldsymbol{g}\right)=$ $\eta_{a n}\left(\boldsymbol{g}_{0}\right)+\eta_{a n}^{\prime} \delta \boldsymbol{g}+\ldots$ and $C_{a n}\left(\boldsymbol{g}_{0}+\delta \boldsymbol{g}\right) \approx C_{a n}\left(\boldsymbol{g}_{0}\right)+C^{\prime} \delta \boldsymbol{g}$. The boundary conditions are $\delta \boldsymbol{f}=0$ Since the velocity shear $\boldsymbol{g}_{0}\left(V, \lambda_{H}\right)$ and $\boldsymbol{f}_{0}\left(V, \lambda_{H}\right)$ are functions of $\lambda_{H}$, this homogeneous system is an eigenvalue problem for $\lambda_{H}$. If such an eigenvalue $\Lambda_{H}$ exists, the non-linear problem has more than one solution and these bifurcate at $\Lambda_{H}$. In general, it is difficult to establish sufficient criteria for the existence of a bifurcation point; it is easier to find necessary criteria. Let us, for the moment, drop the anomalous terms and ask for bifurcation of the classical system. Multiplying with $\delta \boldsymbol{f}$ and integration over $V$ yields

$$
\int\left(\frac{d \delta \boldsymbol{f}}{d V} \cdot \eta_{b} \cdot \frac{d \delta \boldsymbol{f}}{d V}-\delta \boldsymbol{f} \cdot C \cdot \delta \boldsymbol{f}+\frac{1}{2} \delta \boldsymbol{f} \cdot \frac{d G}{d V} \cdot \delta \boldsymbol{f}\right) d V=0
$$

Since $G$ is a diagonal matrix, the derivative is also a diagonal matrix and reads explicitly

$$
\frac{\delta_{e}^{2}}{a^{2}} \frac{d G}{d V}=\frac{\delta_{e}^{2}}{a^{2}}\left\{\frac{m_{k}}{m_{e}} \frac{d F_{k}}{d V} E \delta_{i k}\right\}=\left\{\frac{m_{k}}{m_{e}}<s_{k}>E \delta_{i k}\right\}
$$

If all sources are positive, $d G / d V$ is a positve diagonal matrix and the third term in Eq. (95) is positive. The second term is the power dissipated by Coulomb collisions and is also positive. The first term in Eq. (95) is the power dissipated by viscous forces and since this term is also positive the integral relation leads to a contradiction which implies that a bifuraction cannot occur. From this we infer that only the non-linearity in the anomalous coefficients can cause bifurcations. Using the same method of contradiction we can handle the full system and formulate the following theorem: Let $\boldsymbol{f}_{0}(V, \lambda)$ be a solution of the non-linear problem Eq. (87) in a 
domain $D: \lambda_{0}<\lambda<\lambda_{1}$ and let $\eta_{b}+\eta_{a n}\left(\boldsymbol{g}_{0}\right)+\boldsymbol{g}_{0} \eta_{a n}^{\prime}=\mathbf{A}^{s}+\mathbf{A}^{a}$ be the decomposition into a symmetric and antisymmetric matrix. Furthermore, we make the decomposition

$$
\mathbf{B}^{s}+\mathbf{B}^{a}=C_{a n}\left(\boldsymbol{g}_{0}-\frac{d}{d V} C^{\prime}\left(\boldsymbol{g}_{0}\right) \boldsymbol{f}_{0}\right.
$$

The following statement holds: If $\mathbf{A}^{s}, \mathbf{B}^{s}, d G / d V$ are positive definite matrices, there is no bifurcation point in the domain $D: \lambda_{0}<\lambda<\lambda_{1}$. If these criteria are violated, bifurcation can occur.

This is the generalisation of the conditions (91) to a system. It should be mentioned that the source terms are kept fixed in this bifurcation analysis. If, as a consequence of bifurcation, the plasma exhibits multiple solutions for the poloidal and axial velocities, these will lead to different plasma profiles and different source terms. However, the bifurcation point is a point in parameter space were multiple solutions merge and the source terms are equal.

If a point of bifurcation exists, an expansion technique can be employed to compute the various branches of the solution in the vicinity of the bifurcation point. This technique is useful to find out whether the transition from one branch to another one has a hard onset or a soft onset. However, in solving the problem numerically, a different approach is recommended. In order to convert the second order sytem into a first order system one introduces the transformation

$$
\boldsymbol{g} \mapsto \boldsymbol{z} \quad ; \quad \boldsymbol{z}=\left(\eta_{b}+\frac{\delta_{e}^{2}}{a^{2}} \eta_{a n}(\boldsymbol{g})\right) \boldsymbol{g}
$$

Inverting this system with respect to $\boldsymbol{g}$ yields $\boldsymbol{g}=\boldsymbol{H}(\boldsymbol{z})$ and the first order system is

$$
\begin{aligned}
-\frac{d \boldsymbol{z}}{d V}+\frac{\delta_{e}^{2}}{a^{2}} \frac{d}{d V} G \boldsymbol{f} & =F+\frac{\nu_{e}}{\Omega_{e}}\left(C+C_{a n}(\boldsymbol{H}(\boldsymbol{z})) \boldsymbol{f}+U_{\text {loop }}\right. \\
\frac{d \boldsymbol{f}}{d V} & =\boldsymbol{H}(\boldsymbol{z})
\end{aligned}
$$

If the map $M: \boldsymbol{g} \mapsto \boldsymbol{z}$ is not uniquely invertible, there are several branches $\boldsymbol{H}_{j}(\boldsymbol{z}), j=1,2$. and there are several solutions of the problem. The uniqueness of the inversion depends on the Jacobian of the transformation $M$. If the map $M$ is one-to-one, the anomalous viscosity does not lead to a bifurcation.

\section{The stellarator model}

In the simplest case, the tokamak model has 4 coupled equations, for electrons and ions poloidal and axial rotation. As mentioned above, a stellarator can be modeled without poloidal field and toroidal loop voltage. The magnetic field is a straight field and there is no axial net current. In this case we may set all mean fluxes in the $z$-direction equal to zero, $u_{k}^{t}=0$, and only the poloidal equations are left. If this model is not sufficient, one could make use of the tokamak model with finite poloidal field, but ignore the axial net current and the loop voltage. The equations for the poloidal rotation are

$$
L_{p p, k} u_{k}^{p}-\frac{m_{k}}{m_{e}} \frac{\delta_{e}^{2}}{a^{2}} \frac{d}{d V} \eta_{k}^{p} \frac{d u_{k}^{p}}{d V}+\frac{m_{k}}{m_{e}} \frac{\delta_{e}^{2}}{a^{2}} \frac{d}{d V}\left(F_{k} u_{p, k}\right)=\sigma_{k} F_{k} b^{t}(V)+\frac{\nu_{e}}{\Omega_{e}} \sum_{i}\left(f_{i k}+\gamma_{i k}\right) u_{p, i}
$$

There is a coupling term $L_{p t . k} u_{k}^{p}$ which in general transfers momentum from the poloidal motion to axial motion, however in a straight magnetic field this force is zero; in the tokamak model this off-diagonal term cannot be neglected. The shear viscosity term is explicitly

$$
L_{p p, k} u_{k}^{p}=-\frac{d}{d V}<\boldsymbol{e}_{p} \cdot \pi_{k}^{\star}\left[\boldsymbol{e}_{p}\right] \cdot \nabla V>\frac{d u_{k}^{p}}{d V} \approx-\frac{d}{d V} \eta_{1} V^{2} \frac{d u_{k}^{p}}{d V}
$$


With the help of $\boldsymbol{e}_{p}^{2}=2 V$ and $\boldsymbol{e}_{p}^{2} u^{p}=u_{p}$ we introduce the covariant component which leads to

$$
L_{p p, k} u_{k}^{p}=-\frac{V}{2} \frac{d}{d V} \eta_{1} \frac{d u_{p, k}}{d V}
$$

If there is no feedback of the velocities on the anomalous transport coefficient there is no room for bifurcation and simulation of LH-transition. Let us consider a two-fluid plasma with hydrogen and analyse the relation to the Staebler-Hinton model. For this reason we neglect all viscous and inertial terms on the left hand side and start from the difference of the two equations (100)

$$
F b^{t}(V)=\frac{\nu_{e}}{\Omega_{e}}\left(f_{e i}+\gamma_{e i}\right)\left(u_{p, i}-u_{p, e}\right) \quad ; \quad n_{e}=n_{i}=n ; \quad F_{e}=F_{i}=F
$$

Here we assumed that the matrix $\gamma$ is symmetric which, in general, is not the case. The radial force balance is

$$
\beta_{e}^{\prime}(V)=n \Phi^{\prime}(V)-n b^{t} u_{e}^{p} \quad ; \quad \beta_{i}^{\prime}(V)=-n \frac{d \Phi}{d V}+n b^{t} u_{i}^{p}
$$

Sum and difference of these two equations yield

$$
\beta^{\prime}(V)=n b^{t}\left(u_{p, i}-u_{p, e}\right) \quad ; \quad \frac{1}{2}\left(\beta_{e}^{\prime}(V)-\beta_{i}^{\prime}(V)\right)=n \Phi^{\prime}(V)-n b^{t}\left(u_{p, e}+u_{p, i}\right)
$$

Inserting this into Eq. 103 leads to

$$
F b^{t}(V)=\frac{\nu_{e}}{\Omega_{e}}\left(f_{e i}+\gamma_{e i}\right) \frac{1}{n b^{t}} \beta^{\prime}(V)
$$

The particle flux is proportional to the pressure gradient. The ansatz of Hinton and Staebler is $n^{\prime}$ instead of $\beta^{\prime}(V)$. They propose the anomalous diffusion coefficient

$$
D_{a n}=D_{0}+\frac{D_{1}}{1+\alpha\left(\Phi^{\prime \prime}\right)^{2}}
$$

which accounts for the stabilising effect of the velocity shear, and retain only the ion pressure in the radial balance. The shear of the $\boldsymbol{E} \times \boldsymbol{B}$ velocity is proportional to the density gradient and pressure gradient

$$
\Phi^{\prime \prime}=-\frac{n^{\prime} \beta_{i}^{\prime}}{n^{2}}
$$

Their particle flux is

$$
F=-\left(D_{0}+\frac{D_{1}}{1+\alpha\left(\Phi^{\prime \prime}\right)^{2}}\right) n^{\prime}
$$

Together with Eq. 108, this equation has multiple solutions for $n^{\prime}$. There are two weak points in this derivation. The first one is the ad hoc ansatz of the particle flux being proportional to the density gradient and the second is the approximation of the radial force balance.

A better method would be to start from Eq. (103) as it is and insert $D_{a n}$ for $\gamma_{e i}$. In the special case $T_{e}=T_{i}$ we get $\beta_{i}=\beta_{e}$ and the radial force balance leads to

$$
\Phi^{\prime \prime}(V)=b^{t} \frac{d}{d V}\left(u_{e}^{p}+u_{i}^{p}\right)
$$

The shear of the electric drift is equal to the velocity shear of electrons plus the shear of ions. The pressure gradient is proportional to the difference of the poloidal velocities of electrons and ions (diamagnetic current) and the radial electric field is proportional to the sum of these 
velocities. Next, the viscous and inertial terms are included and the electron and ion equations are

$$
\begin{aligned}
\frac{m_{i}}{m_{e}} \frac{\delta_{e}^{2}}{a^{2}} \frac{d}{d V} e_{p}^{2} \eta_{i}^{p} \frac{d u_{i}^{p}}{d V}+\frac{m_{i}}{m_{e}} \frac{\delta_{e}^{2}}{a^{2}} \frac{d}{d V}\left(F u_{p, i}\right) & =F b^{t}(V)+\frac{\nu_{e}}{\Omega_{e}}\left(f_{e i}+D_{a n}\right)\left(u_{p, i}-u_{p, e}\right) \\
\frac{\delta_{e}^{2}}{a^{2}} \frac{d}{d V} e_{p}^{2} \eta_{e}^{p} \frac{d u_{e}^{p}}{d V}+\frac{\delta_{e}^{2}}{a^{2}} \frac{d}{d V}\left(F u_{p, e}\right) & =-F b^{t}(V)-\frac{\nu_{e}}{\Omega_{e}}\left(f_{e i}+D_{a n}\right)\left(u_{p, i}-u_{p, e}\right)
\end{aligned}
$$

We have assumed that the eddy viscosity is much larger than the collisional shear viscosity. Since the radial mixing length of turbulent eddies is larger than the gyro radius, eddy viscosity can easily provide the main momentum transport. If the anomalous coefficient $D_{a n}$ is larger than the classical friction coefficient we may neglect $f_{e i}$. In the Staebler-Hinton ansatz of $D_{a n}$, we insert the velocity shear of electrons and ions as given in Eq. (110). One could adopt the same ansatz for the eddy viscosity which would make these a decreasing function of the velocity shear. Whatever ansatz or model is used, the sum of the right hand sides is always zero, which means that the sum of the left hand sides is also zero. The sum can be integrated once leading to

$$
\frac{m_{i}}{m_{e}}\left(\boldsymbol{e}_{p}^{2} \eta_{i}^{p} \frac{d u_{i}^{p}}{d V}+F u_{p, i}\right)+\left(\boldsymbol{e}_{p}^{2} \eta_{e}^{p} \frac{d u_{e}^{p}}{d V}+F u_{p, e}\right)=C
$$

The constant $C$ is zero since the left hand side tends to zero at $V \longrightarrow 0$. At this point the large mass difference of the particles comes into play. Since the two terms on the left are equal for any solution this implies that the electron velocity is much larger than the ion velocity. However, here the boundary conditions are important: At fixed boundary conditions the large ion eddy viscosity will slow down the ions to a small value - independently of the sign of the eddy viscosity. Under free boundary conditions there would be no viscous dissipation if the ion flow is a rigid rotation. In the extreme case of nearly zero ion velocity we may neglect the coupling between the two equations and restrict our attention to the electron equation. In this case the plasma is confined by the diamagnetic flow of the electrons and the key for transport barriers lies in the poloidal velocity of the electrons. The ion pressure is balanced by the radial electric field. The particle flux $F$ occurs twice in each equation and because of the smallness of $\delta_{e}^{2} / a^{2}$ we may neglect the first term with $F$ and write the electron equation as

$$
\frac{\delta_{e}^{2}}{a^{2}} \frac{d}{d V} e_{p}^{2} \eta_{e}^{p} \frac{d u_{e}^{p}}{d V}=-F(V) b^{t}(V)+\frac{\nu_{e}}{\Omega_{e}}\left(f_{e i}+D_{a n}\right) u_{p, e}
$$

This is just the type of equation which has been investigated with respect to bifurcation in the previous section.

\section{$6 \quad$ Numerical results}

The computations presented in the following simulate the experimental results found in Wendelstein 7-AS [13]. The density in the HDH-regime reaches $4 * 10^{20} \mathrm{~m}^{-3}$, while the temperature stays in the range of $500 \mathrm{eV}$ leaving the plasma in the Pfirsch-Schlüter regime or close to the plateau regime. For this reason neoclassical effects are negligible and the fluid model provides an adequate description. The cylindrical model discussed here neglects all toroidal effects, however, as the main purpose is to describe the transition from a peaked density profile to a flat density profile with steep gradients at the edge. The cylindrical model does not explain why this phenomenon occurs only at some specific values of the rotational transform. The temperature in the experiment is nearly constant during this transition and therefore only the momentum balance is solved in the following numerical computations. The HDH-mode is a quiescent phase 
without any significant fluctuations in the plasma. Another important feature of the HDH-mode is the strong reduction of impurity influx. In the following computations, we do not propose a specific model of the anomalous transport coefficients. The only assumption is that the transport coefficients are bounded from below and above. The Hinton-Staebler model falls into this category since we get $D_{0} \leq D \leq D_{0}+D_{1}$. The purpose of the computations is to study how the plasma profiles change when the anomalous transport coefficients adopt the maximum or minimum values. In this procedure the equations are linear and can be solved by difference methods.

The source function is given by $S_{e}=S_{H}=<\sigma v>_{\text {ion }} n(x) n_{0}(x) ; n_{e}=n_{H}=n(x)$ is the electron density and $n_{0}$ the density of neutrals. $\langle\sigma v\rangle_{i o n}$ is the ionisation rate by electron collisions. Here, we describe the profile of the neutrals by an exponential function

$$
n_{0}(x)=n_{0}(1) \exp \left[-K \int_{x}^{1} n(x) d x\right]
$$

which provides us with an approximation of the source function

$$
s(x)=K_{0} K n(x) \exp \left[-K \int_{x}^{1} n(x) d x\right]
$$

$L=1 / K$ is the non-dimensional decay length of the neutrals and $K_{0}$ is proportional to the neutral gas flux. Electron and ion fluxes are equal $\left(F_{e}=F_{H}=F\right)$. The poloidal electron equation is

$$
-b(x) \frac{F}{x}+\frac{1}{x} \frac{\nu_{e e}}{\Omega_{e}} f(x)\left(u_{e}-u_{H}\right)+4 \frac{d}{d x} \eta_{e, e f f} \frac{d u_{e}}{d x}-2 \frac{\delta_{e}^{2}}{a^{2}} \frac{1}{x} \frac{d F u_{e}}{d x}=0
$$

and the ion equation

$$
b(x) \frac{F}{x}+\frac{1}{x} \frac{\nu_{e e}}{\Omega_{e}} f(x)\left(u_{H}-u_{e}\right)+4 \frac{d}{d x} \eta_{H, \text { eff }} \frac{d u_{k}}{d x}-2 \frac{m_{H}}{m_{e}} \frac{\delta_{e}^{2}}{a^{2}} \frac{1}{x} \frac{d F u_{H}}{d x}=0
$$

with

$$
f(x)=\frac{n^{2}(x)}{T^{1.5}(x)}+C_{a n}
$$

in non-dimensional units. $C_{a n}$ is the anomalous friction coefficient. The reference values in the numerical calculations are $B_{0}=2.5 \mathrm{~T}, n_{0}=10^{20} \mathrm{~m}^{-3}$ and $T_{0}=1 \mathrm{keV}$. The minor radius of the plasma in W 7-AS is about $a=0.15 \mathrm{~m}$. The non-dimensional parameters are

$$
\frac{\nu_{e e}}{\Omega_{e}}=3.37 \times 10^{-7} \quad ; \frac{\delta_{e}^{2}}{a^{2}}=1.25 \times 10^{-5}
$$

The beta profile is computed by integrating the radial force balance

$$
-\frac{d \beta}{d r}=\frac{b(r)}{r} n(r)\left(u_{e}-u_{H}\right)
$$

At low beta we may neglect the diamagnetic drop of the magnetic field and consider $b(r)$ as constant;at large beta this effect is taken into account. The numerical calculations proceed as follows: given a lowest order guess of the density profile we compute the radial flux

$$
F=\int_{0}^{x} s(x) d x
$$

with a given source function $s$ (Eq. 115). Solving the coupled system Eqs. (116) and (117) yields the velocities $u_{e}$ and $u_{H}$. In a next step a beta profile and is computed in Eq. (120) and 
with the help of the temperature a new density profile. Iterating this procedure provides us with the self-consistent profiles $n(r), u_{e}(r), u_{i}, \beta(r)$. The temperature is fixed in all computations; the figure Fig. (1) shows the profile.

The boundary conditions are $u_{e}=0 ; u_{i}=0$ at $x=0$ and $x=1$. The anomalous viscosity is described in section (4). In the numerical calculation we model the viscosity as follows

$$
\eta_{e, e f f}=\frac{\nu_{e e}}{\Omega_{e}} \eta_{e} \quad ; \quad \eta_{H, e f f}=\frac{m_{H}}{m_{e}} \frac{\nu_{e e}}{\Omega_{e}} \eta_{i}
$$

Here, $\eta_{e}$ and $\eta_{i}$ are constant This is the simplest approximation to the anomalous visccosity. Setting $\eta_{e}=1$ and $\eta_{i}=1$ means that the electron viscosity has about the same effect as the classical electron-ion Coulomb interaction. Because of the mass ratio the ion viscosity is large and inhibits any ion rotation. This implies that the ion pressure is confined by the radial electric field and the electron diamagnetic flow confines the sum of electron and ion pressure. Note, that zero ion rotation does not mean zero electric field.

Reducing the electron viscosity has the strongest impact on the poloidal velocity of the electrons. Since the ion viscosity is much larger than the electron viscosity $\left(m_{H} / m_{e}=1836\right)$ the poloidal ion velocity is almost zero; the diamagnetic current is totally carried by the electrons. For this reason, any change of the electron poloidal velocity has a strong impact on the plasma confinement.

\subsection{HDH-mode}

Detailed results of the HDH-mode in Wendelstein 7-AS have been published in [15]. The density is in the range of $1.5 \times 10^{20} \mathrm{~m}^{-3}$ and the temperature less than $360 \mathrm{eV}$. The HDH-mode in Wendelstein 7 -AS is characterized by a flat density profile and a steep density gradient in the boundary region. While in "normal" confinement the density peaks in the centre, cold gas puffing leads to a transition to the HDH-mode. Suppression of turbulence is a further important feature of the HDH-mode, indicating that the improvement of the confinement may be correlated to a decrease of turbulent transport.

There are some features of the HDH-mode which the present model is not able to explain. In particular, dependence on the external rotational transform can only be understood in a toroidal model taking into account the neoclassical viscosity and it variation with rotational transform. Another outstanding property of the $\mathrm{HDH}$-mode is its resistance against impurity influx. Although the model discussed above holds for a multi-species plasma with impurity ions, the numerical analysis has been focussed on a two-component plasma only. The code will be extended to impurities in future.

In order to simulate the transition of the density profiles, two parameters are changed: cold gas flux will be increased and the anomalous electron viscosity will be reduced. Ion viscosity is large and kept fixed. As long as $\eta_{i}$ is of the order unity, the ion velocity is close to zero and any changes of the ion viscosity has negligible effect on the confinement.

The first example assumes that the friction between electrons and ions is classical; anomalous effects occur in electron and ion viscosity. Reducing the electron viscosity while the gas influx is increased leads to a flat density profile. The input parameters are: Case 1: $\eta_{e}=1.0, \eta_{i}=$ $1.0, K_{0}=0.7$, case $2 ; \eta_{e}=0.5, \eta_{i}=1.0, K_{0}=1.3$. The two cases differ only by a factor two: the electron viscosity is diminuished by a factor two and the cold gas input grows by less than a factor two. The density at the plasma centre is about the same in both cases. The following figure shows a fit to the temperature profile measured in the HDH-mode in Wendelstein 7-AS. This profile will be kept fixed in the subsequent computations. 

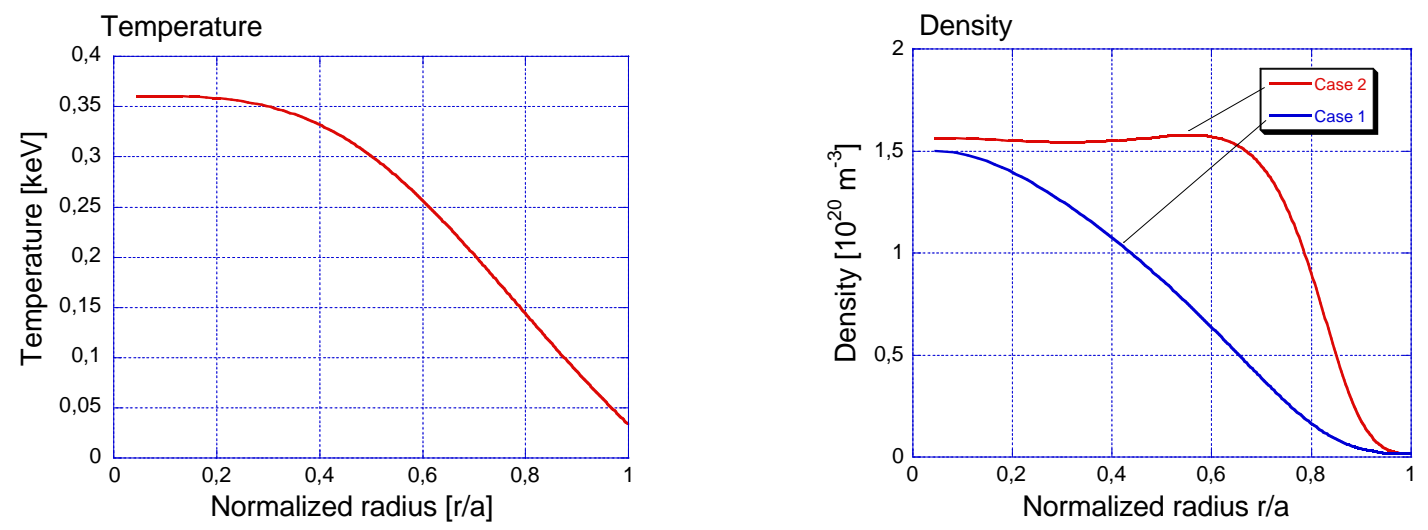

Figure 1: Left: Temperature profile. Right: Density profile.
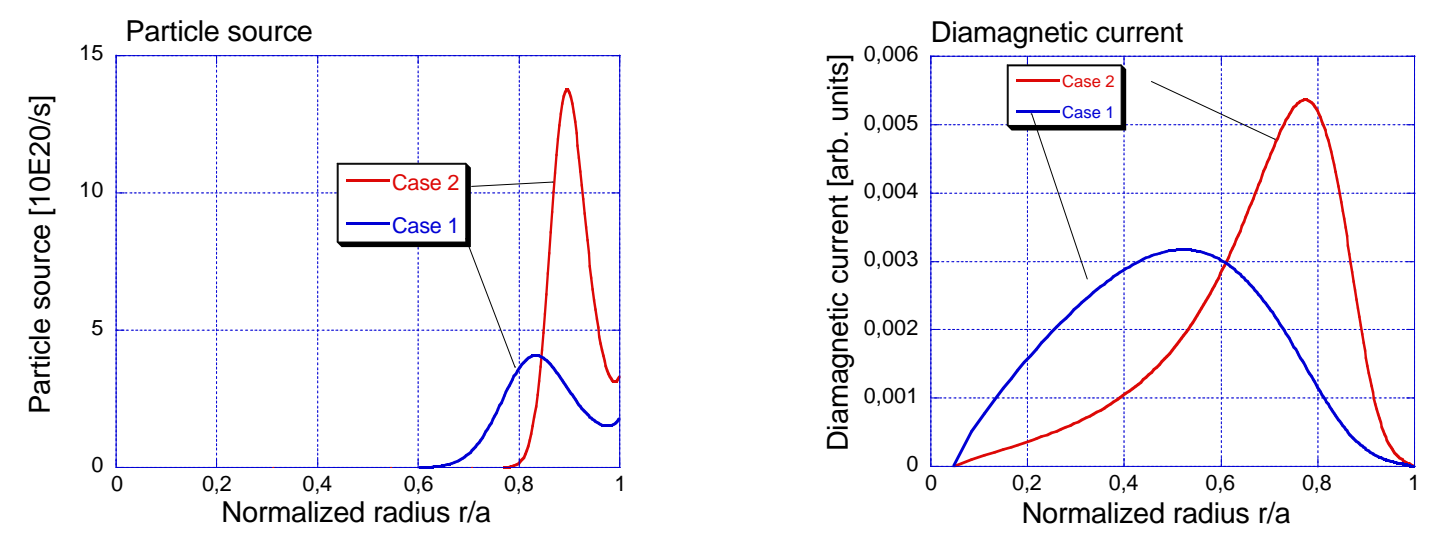

Figure 2: Left: Plasma source function. Right: Diamagnetic current.
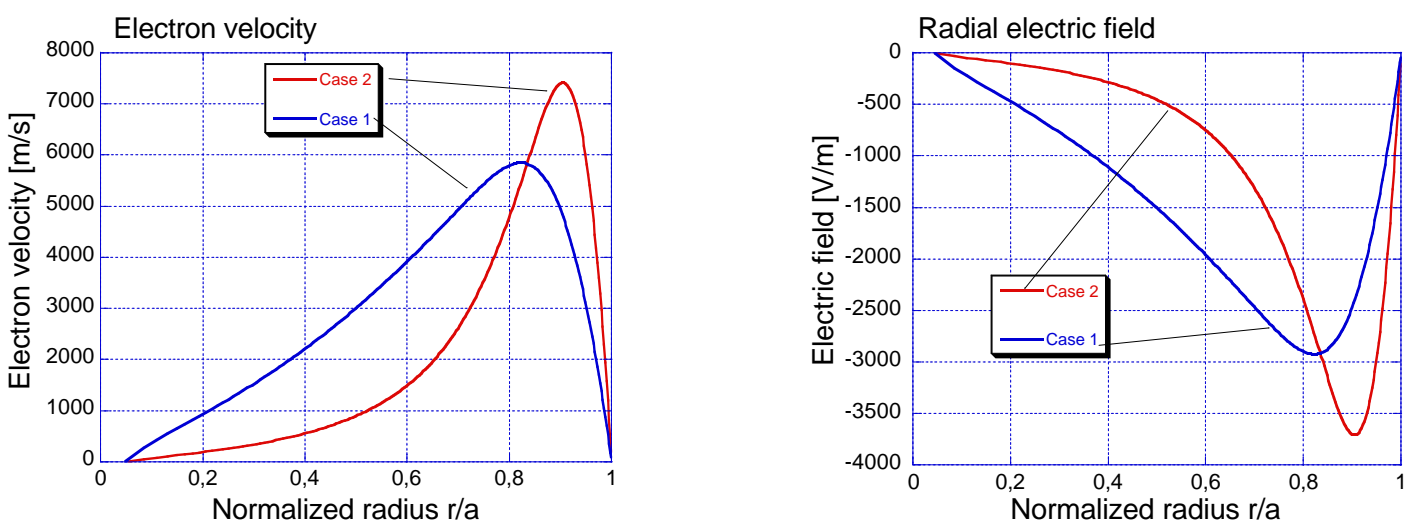

Figure 3: Left: Poloidal electron velocity . Right: Radial electric field.

The basic features of the HDH-mode are reproduced in this example. The density exhibits a steep gradient in the boundary region, the electric field increases in this region, the plasma source function is shifted towards the boundary and the diamagnetic current density grows thus compensating the steep pressure gradient.

In the next example the condition is to keep the cold gas input nearly fixed and to vary the transport coefficients only. There is only a slight increase of the gas flux from $K_{0}=1.3$ to $K_{0}=1.4$. The electron viscosity will be changed from $\eta_{e}=1.2$ to $\eta_{e}=0.255$. Furthermore, the 
anomalous friction between electrons and ions will be reduced by factor of ten. The anomalous friction is described by a coefficient $C_{a n}$ which is added to the coefficient $f(x)$ in Eq. (118). This coefficient is a constant in the following example which, in general, may not be the case.
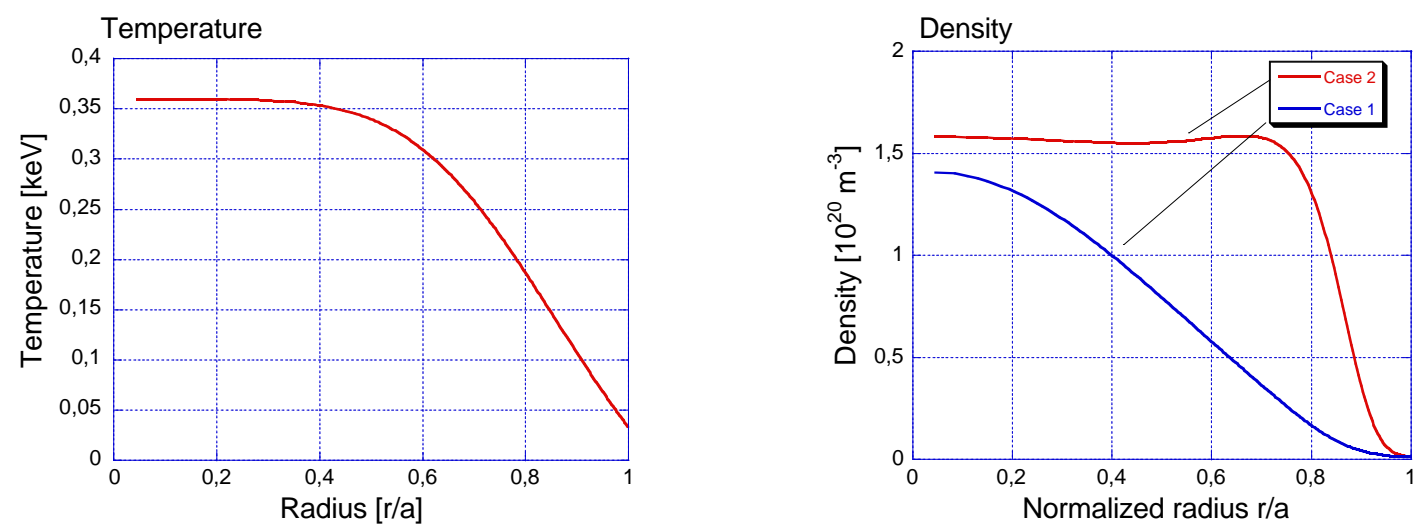

Figure 4: Left: Temerature profile. Right: Density. Case 1: $\eta_{e}=1.2, \eta_{i}=1.0, K_{0}=1.3$, $C_{a n}=10.9$. Case $2 \eta_{e}=0.255, \eta_{i}=1.0, K_{0}=1.4, C_{a n}=1.0$
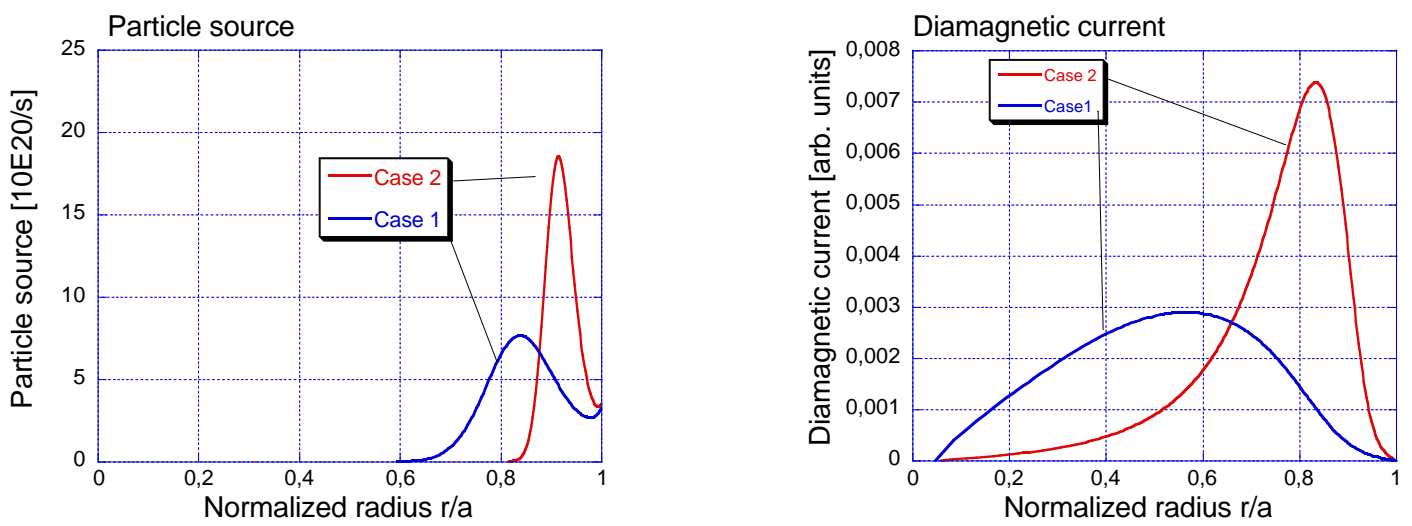

Figure 5: Left: Plasma source function. Right: Diamagnetic current.

The last example shows that the reduction of two anomalous coefficients, the electron viscosity and the anomalous electron-ion friction is sufficient to shape the peaked density profile into a flat density profile with the same central density. Here, the anomalous friction coefficient is constant, however this approximation is sufficient to model the basic features of the HDH-mode.

Reducing the anomalous coefficent leads to a better confinement. In the last example the particle confinement time grows from $\tau_{p}=13.7 \mathrm{~ms}$ to $\tau_{p}=31.4 \mathrm{~ms}$. These numbers depend on the choice of the effective ionisation rate $K_{0}$ and their absolute values may not be correct. However, the growth by a factor 2.3 is correct and fits to the experimental results. Since we kept the temperature constant in these simulations, the energy confinement time grows by the same factor.

In the case of rigid boundaries the ion rotation is rather small since eddy viscosty provides a strong momentum loss to the boundary. This is not the case when we impose the free boundary conditions. There is no momentum loss to the boundary but eddy viscosity will lead to rigid rotation without velocity shear. In this case we infer from Eq. (112) that the ion poloidal velocity is smaller than the electron velocity by the factor $m_{e} / M_{i}$. The mass ratio determines the relation. 


\section{Conclusions}

In this paper we have investigated the Reynolds averaged momentum balance in a plasma cylinder. The general ansatz includes an arbitrary number of particle species, however the numerical calculations have been restricted on a two-component plasma. In a cylindrical model neoclassical effects are small and negligible. However, the turbulence driven by gradient driven instabilities introduces some new effects which may also be relevant in toroidal plasmas. In particular, turbulent shear viscosity or eddy viscosity shapes the poloidal velocity of the plasma constituents and the diamagnetic current. Turbulent shear viscosity contributes to anomalous plasma losses as does the enhanced friction induced by turbulence. Including shear viscosity leads to second order differential equations for the poloidal velocity and as a consequence the linear and algebraic relation between radial fluxes and gradients is no longer valid. In a turbulent plasma where eddy viscosity and anomalous friction govern the poloidal velocity profile, Fick's law does not hold.

The radial electric field only occurs in the radial momentum balance; the poloidal momentum balance is not affected by the electric field. As shown, the electric potential can be eliminated and after the poloidal momentum balance has been solved the electric field is computed as the last step. If the ion diamagnetic current has been slowed down to zero the ion pressure gradient is balanced by the radial electric field. This example is shown in the numerical calculations.

The eddy viscosity is the effect of turbulent inertial forces and because of the larger mass, ion viscosity is much larger than electron viscosity. In case of Dirichlet boundary conditions (zero poloidal velocity at the boundary) large ion viscosity slows down the diamagnetic ion current to nearly zero and the plasma is mainly confined by the electron diamagnetic current. In this case plasma confinement depends on the anomalous effects shaping the radial profile of the poloidal electron velocity.

In the classical model without shear viscosity boundary conditions on the poloidal velocity are not required. However, solving a differential equation of second order needs boundary conditions. These boundary conditions reflect the physics at the boundary, which can either be a limiter, a material wall or a last magnetic surface without contact to material obstacles.

The dependence of the anomalous viscosity on the velocity shear can give rise to multiple solutions and bifurcation. Linearisinig the non-linear eqquations allows one to determine the bifurcation point and to formulate necesary conditions for bifurcation. The relation to the Stabler-Hinton model has been discussed and it can be shown that a threshold in cold gas refueling exists below which the bifurcation does not occur.

Since the aim of the numerical calculations was to simulate some features of the HDH-mode in Wendelstein 7-AS, the energy balance has been neglected in this paper. The temperature remains nearly unchanged in the transition to the $\mathrm{HDH}$-mode. In general, however, transition to $\mathrm{H}$-mode depends on the heating power, and therefore the energy balance will be taken into account in future investigations.

As the main result, we found the important role of the anomalous viscosity. Reducing the turbulence, anomalous shear viscosity and anomalous friction coefficients lead to an increase of the diamagnetic current and a steepening of the plasma pressure gradient. Furthermore, the reduction of the viscosity shifts the peak of the diamagnetic current towards the plasma boundary and thus to steeper pressure profiles in the edge region. For simplicity we have assumed that the anomalous viscosity is a constant and independent of the radial position. Localizing anomaly to the edge region would enhance the effect. Therefore, the formation of a transport barrier can be understood as a reduction of the anomalous shear viscosity accompanied by a shift of the diamagnetic current towards the plasma edge. Since the sum of the poloidal viscous forces is zero, the poloidal ion velocity of a two-component plasma is small and negligible if the ion viscosity is more than 10 times larger than the electron viscosity. The radial electric field is determined 
by the ion pressure gradient. Steepening the pressure gradient also leads to a steepening of the electric field and its gradients.

What is the physics behind the formation of a transport barrier? Since the viscosity reduces the poloidal velocity and thus the diamagnetic current, any reduction of the viscosity increases the diamagnetic current and improves the confinement. At constant gas input, this effect leads to a growth of the density. With growing density, the particle source and the radial particle flux are shifted towards the plasma edge. Since the radial particle flux times the magnetic field is the driving force of the poloidal rotation, this implies that the driving force is also shifted towards the plasma edge and thus localizes the diamagnetic current more and more to the edge region.

\section{References}

[1] Hinton F L and Staebler G M 1993 Phys. Fluids B 51281

[2] Groebner R J, Mahdavi M A, Leonard A W, Osborne T H, Wolf N S, Poerter G D, Stangeby P C, Brooks N S, Colchin R J and Owen L W 2004 Nucl. Fusion 44 204- 213

[3] Connor J W and Wilson H R, Plasma Phys. Control. Fusion 42 (2000), R1-R74

[4] Coronado M and Wobig H 1986 Phys Fluids 29527

[5] Wobig H and Kisslinger J ,Plasma Phys. and Control. Fusion 33, (2000) 823-841

[6] Stringer T A , Phys. Rev. Lett., 22 ,p. 1770 (1969)

[7] Lugt H J, Vortex Flow in Nature and Technology, John Wiley\&Sons, New York 1983

[8] Wobig H, Plasma Phys. and Control. Fusion, 38, (1996) 1053-1081

[9] Biglari H, Diamond P H and Terry P W 1990 Phys. Fluids B2 1

[10] Diamond P H, Itoh K, Itoh S-I and Hahm T S $200420^{\text {th }}$ IAEA Fusion Energy Conference, paper $\mathrm{OV} / 2-1$

[11] Kaladze T D, Wu D J, Pokhotelov, Sagdeev R Z, Stenflo L and Shukla P K 2005 Phys. Plasmas 12122311

[12] Grigull P et al., Plasma Phys. Control. Fusion 43 (2001) A175

[13] McCormick K et al., Phys. Rev. Letters 89 (2002) . p. 152

[14] Wagner F et al. 2005 Phys. Plasmas 12072509

[15] McCormick K et al. Proc. Int. Stellarator Workshop, Greifswald 2003

[16] Hirshman S, Sigmar D, Nuclear Fusion 21,(1981) p. 1080-1198

[17] Braginskii S I, Rev. of Plasma Phys. Vol. I, p. 250, Consultants Bureau New York 1965

[18] Stacey W M Jr. and Sigmar D 1985 Phys. Fluids 28 (9) 2800

[19] Claassen H A, Gerhauser H, Rogister A and Yarim C, 2000 Phys. Plasmas 73699

[20] Ramos J J, 2005 Phys. Plasmas 12112301

[21] Mikhailovskii A B and Tsypin V S, 1985 Beitr. Plasmaphys. 24335 
[22] Spada M and Wobig H 1992 J. Phys. A: Math. Gen. 251575

[23] Doering C R and Gibbon J D, Applied Analysis of Navier-Stokes Equations, Cambridge University Press, 1995

[24] Coronado M and Galindo Trejo J 1990 Phys Fluids B 2350

[25] Nührenberg J and Zille R 1988 Phys. Lett. 128A 113

[26] Grieger G et al. 1992 Phys. Fluids B4 2081

[27] Wobig H and Kisslinger J, Plasma Phys. and Control. Fusion, 33, (1995) 893-922

[28] Wobig H, Plasma Phys. and Control. Fusion, 41, (1999) A159

[29] Gatski T B, Hussaini M Y, Lumley J L, Simulation and Modeling of Turbulent Flows Oxford University Press 1996, New York, Oxford

[30] Terry P W, 2000 Rev. Mod. Phys. 721 109-165

[31] Hassam A B and Drake J F, 1993 Phys. Fluids B5 4022

[32] Mayr H G, Harris I, Schatten K H, Stevens-Rayburn D R and Shan K L, Earth, Moon and Planets 41, (1988), 45.

[33] Tennekes H and Lumley J L A First Course in Turbulence MIT Cambridge MA, 1972

[34] Kim Eun-jin, Hahm T S and Diamond P H Physics of Plasmas 8(8), 3576-3582 (2001).

[35] Sugama H and Horton W 1997 Phys. Plasmas 4 (2) 405

[36] Shaing K C, Lee G S, Carreras B A, Houlberg W A, Crume E C Proc. of the 12th Int. Conf. on Plasma Physics and Controlled Nuclear Fusion Research Nice 1988, IAEA Vienna 1989, Vol. 2, p 13

[37] Shaing K C, 1994 Phys Plasmas 1219

[38] Staebler G M and Dominguez R R 1993 Nuclear Fusion 33177

[39] Staebler G M, 2004 Phys. Plasmas 111064

[40] Balescu R and Fantechi S, 1990 Phys. Fluids B2 2091

[41] Shaing K C 1988 Phys. Fluids 318 2249-2265

[42] Shaing K C, 2001 Phys Plasmas 8193

[43] Keller J B and Antman S Bifurcation Theory and Nonlinear Eigenvalue Problems W. A. Benjamin, Inc. New York 1969

[44] Hassam A B and Kulsrud R M 1978, Phys. Fluids 21, 2271

[45] Staebler G M, Hinton F L, Wiley J C, Dominguez R R, Greenfield C M, Gohil P KurkiSuonio T K and Osborne T H 1993 Phys. Plasmas 1 (4) 909

[46] Hassam A B, Antonsen M, Drake J F and Liu C S 1991, Phys. Rev. Letters 66, No.3, 309

[47] Peeters A G, Phys. Plasmas , 5 (1998), p. 2399 
[48] Ramos J J, 2005 Phys. Plasmas 12112301

[49] Shaing K C, Hirshman S P and Callen J D 1994 Phys. Fluids 29521

[50] Gatski T B,Speziale C G, J. Fluid Mech. 254 p. 59-78

[51] Beidler C D, Igitkhanov Y L and Wobig H, Fusion Science and Technology, 46, 2004, p. 64

[52] Guzdar P N, Drake J F, McCarthy D, Hassam A B and Liu C S 1993, Phys. Fluids B5, 3712

[53] Garcia L, Carreras B A, Lynch V E and Diamond P H 1992, Proc. $14^{\text {th }}$ Int Conf. on Plasma Phys. and Contr. Nuclear Fusion Research, Würzburg, 1992, paper CN-56/D-4-6

[54] Schubauer G B and Tchen C M, Turbulent Flow, Princeton University Press, 1961 